CERN-PH-TH/2007-208

LA-UR-07-7323

MIT-CTP-3889

\title{
Quantum Resonant Leptogenesis and Minimal Lepton Flavour Violation
}

\author{
Vincenzo Cirigliano ${ }^{\mathrm{a}}$, Andrea De Simone ${ }^{\mathrm{b}}$, Gino Isidori ${ }^{\mathrm{c}}$, Isabella Masina ${ }^{\mathrm{d}}$ and Antonio Riotto ${ }^{\mathrm{d}, \mathrm{e}}$ \\ a Theoretical Division, Los Alamos National Laboratory, Los Alamos NM 87545, USA \\ b Center for Theoretical Physics, Massachusetts Institute of Technology, \\ Cambridge, MA 02139, USA \\ c Scuola Normale Superiore, Piazza dei Cavalieri 7, I-56100 Pisa, Italy, \\ and INFN, Laboratori Nazionali di Frascati, Via E. Fermi 40, I-00044 Frascati, Italy \\ d CERN, Department of Physics, Theory Division, CH-1211 Geneva 23, Switzerland \\ e INFN, Sezione di Padova, via Marzolo 8, 35131 Padova, Italy
}

Electronic addresses: cirigliano@lanl.gov, andreads@mit.edu, Gino.Isidori@lnf.infn.it, imasina@mail.cern.ch, riotto@mail.cern.ch

\begin{abstract}
It has been recently shown that the quantum Boltzmann equations may be relevant for the leptogenesis scenario. In particular, they lead to a time-dependent CP asymmetry which depends upon the previous dynamics of the system. This memory effect in the $\mathrm{CP}$ asymmetry is particularly important in resonant leptogenesis where the asymmetry is generated by the decays of nearly mass-degenerate right-handed neutrinos. We study the impact of the non-trivial time evolution of the CP asymmetry in the socalled Minimal Lepton Flavour Violation framework where the charged-lepton and the neutrino Yukawa couplings are the only irreducible sources of lepton-flavour symmetry breaking and resonant leptogenesis is achieved. We show that significant quantitative differences arise with respect to the case in which the time dependence of the $\mathrm{CP}$ asymmetry is neglected.
\end{abstract}




\section{Introduction}

The observed baryon number asymmetry of the Universe $\eta_{B} \equiv n_{B} / n_{\gamma}=(6.3 \pm 0.3) \times 10^{-10}$ [1] can be explained by the mechanism of thermal leptogenesis 2, 3, 4, the simplest implementation of this mechanism being realised by adding to the Standard Model (SM) three heavy right-handed $(\mathrm{RH})$ neutrinos, i.e. in the framework of the seesaw [5]. In thermal leptogenesis the heavy $\mathrm{RH}$ neutrinos are produced by thermal scatterings after inflation and subsequently decay outof-equilibrium in a lepton number and CP-violating way, thus satisfying Sakharov's constraints [6, 7]. The dynamically generated lepton asymmetry is then converted into a baryon asymmetry due to $(B+L)$-violating sphaleron interactions [8].

If RH neutrinos are hierarchical in mass, successful leptogenesis requires that the RH neutrinos are heavier than about $10^{9} \mathrm{GeV}$ 9. Hence, the required reheating temperature after inflation cannot be much lower [3, 10, 11, 12]. In supersymmetric scenarios this may be in conflict with the upper bound on the reheating temperature necessary to avoid the overproduction of gravitinos during reheating [13]. In the resonant leptogenesis scenario [14] this tension may be avoided. If the $\mathrm{RH}$ neutrinos are nearly degenerate in mass, self-energy contributions to the $\mathrm{CP}$ asymmetries may be resonantly enhanced, thus making thermal leptogenesis viable at temperatures as low as the $\mathrm{TeV}$. Resonant leptogenesis seems therefore a natural possibility.

Nearly degenerate RH neutrinos naturally arise in the context of models satisfying the hypothesis of Minimal Flavour Violation (MFV) 15, 16, 17. In the quark sector, where the MFV hypothesis has been formulated first, the MFV ansatz states that the two quark Yukawa couplings are the only irreducible breaking sources of the flavour-symmetry group defined by the gaugekinetic Lagrangian [16]. In generic models satisfying this hypothesis, quark Flavour Changing Neutral Currents (FCNC) are naturally suppressed to a level comparable to experiments and new degrees of freedom can naturally appear in the $\mathrm{TeV}$ range. The extension of the MFV hypothesis to the lepton sector (MLFV) has been formulated in Ref. [17] (and further studied in Refs. [18, 19, 20]), where a number of possible scenarios, depending on the field content of the theory, have been identified. The case more similar to the quark sector and more interesting from the point of view of leptogenesis is the so-called extended field content scenario of Ref. [17]. Here the lepton field content is extended by three heavy RH neutrinos with degenerate masses at the tree level. The largest lepton-flavour symmetry group of the gauge-kinetic term is $G_{\max }=S U(3)_{\ell} \times S U(3)_{e} \times O(3)_{N}$ and, according to the MLFV hypothesis, it is assumed that this group is broken only by the charged-lepton and neutrino Yukawa couplings $\lambda_{e}$ and $\lambda_{\nu}$. In relation to leptogenesis, the key feature of this scenario is that the degeneracy of the RH neutrinos is lifted only by corrections induced by the Yukawa couplings, so that we end up with a highly constrained version of resonant leptogenesis. Within this set up, the viability of leptogenesis has first been considered in the one-flavour approximation [21] and subsequently in the flavoured case [22, 23].

Resonant leptogenesis in models satisfying the MLFV hypothesis is the subject of the present paper. Our analysis turns out to be necessary in view of the recent results achieved by studying the dynamics of thermal leptogenesis by means of quantum Boltzmann equations [24] (for an earlier study, see Ref. 25]). Quantum Boltzmann equations were obtained starting from the non-equilibrium quantum field theory based on the Closed Time-Path formulation and have an obvious interpretation in terms of gain and loss processes. However, they differ from the classical Boltzmann equations since there appear integrals over the time where theta functions ensure that the dynamics is causal. In the classical kinetic theory the scattering terms do not include any integral over the past history of the system which is equivalent to assume that any collision in the 
plasma does not depend upon the previous ones. On the contrary, quantum distributions possess strong memory effects and the thermalization time obtained from the quantum transport theory may be substantially longer than the one obtained from the classical kinetic theory. Furthermore, and more importantly, the CP asymmetry turns out to be a function of time, its value at a given instant depending upon the previous history of the system. If the time variation of the $\mathrm{CP}$ asymmetry is shorter than the relaxation time of the particles abundances, the solutions to the quantum and the classical Boltzmann equations are expected to differ only by terms of the order of the ratio of the time-scale of the CP asymmetry to the relaxation time-scale of the distribution. In thermal leptogenesis with hierarchical RH neutrinos this is typically the case. However, in the resonant leptogenesis scenario, $\mathrm{RH}$ neutrinos are almost degenerate in mass and the CP asymmetry from the decay of the $i$-th RH neutrino $N_{i}$ is resonantly enhanced by the $j$-th neutrino if the mass difference $\left(M_{j}-M_{i}\right)$ is of the order of the decay rate of the $\mathrm{RH}$ neutrinos. The typical time-scale to build up coherently the time-dependent CP asymmetry is of the order of $\left(M_{j}-M_{i}\right)^{-1}[24,26$, which can be larger than the time-scale for the change of the abundance of the RH neutrinos. This tells us that in the case of resonant leptogenesis significant differences are expected between the classical and the quantum approach.

In this paper we perform a detailed study of the role of quantum memory effects in the resonant leptogenesis scenario within the MLFV hypothesis showing that memory effects may substantially change the prediction for the baryon asymmetry. We consider both the normal hierarchical $(\mathrm{NH})$ and the inverse hierarchical $(\mathrm{IH})$ cases for light neutrinos and also consider the role played by flavour in leptogenesis [27, 28, 29, 30].

Our analysis is organized as follows. In Section 2 we provide a brief summary of thermal leptogenesis, focussing on the impact of the quantum memory effects. Section 3 contains the key ingredients of the MLFV scenario. In Section 4 we set the stage for the study of the relevance of non-equilibrium quantum effects in MLFV-leptogenesis. We then proceed discussing the impact of quantum effects on the two scenarios in which $\mathrm{CP}$ violation arises from the $\mathrm{RH}$ sector in Section 5, and only from the PMNS matrix in Section 6. We give our conclusions in Section 7 .

\section{A brief summary of thermal leptogenesis}

In order to set the stage for the subsequent analysis about the impact of non-equilibrium quantum effects in the MLFV framework, here we first briefly recall the general features of the thermal leptogenesis scenario, and later discuss the restrictions imposed by the hypothesis of Minimal Flavour Violation.

We consider a model where three right-handed $(\mathrm{RH})$ neutrinos $N_{i}(i=1,2,3)$ with Majorana masses $M_{3} \geq M_{2} \geq M_{1}$ and Yukawa couplings $\lambda_{\nu}$ are added to the Standard Model (SM) Lagrangian. Working in the basis in which the Yukawa couplings for the charged leptons $\lambda_{e}$ are diagonal, the Lagrangian density is given by

$$
\mathcal{L}=\mathcal{L}_{\mathrm{SM}}+\left(\frac{1}{2} M_{i} N_{i}^{2}+N_{i}\left(\lambda_{\nu}\right)_{i \alpha} \ell_{\alpha} H+\bar{e}_{\alpha}\left(\lambda_{e}\right)_{\alpha} \ell_{\alpha} H^{c}+\text { h.c. }\right)
$$

where $\ell_{\alpha}$ and $e_{\alpha}$ indicate the lepton doublet and singlet with flavour $(\alpha=e, \mu, \tau)$ respectively, and $H$ is the Higgs doublet whose neutral component has a vacuum expectation value equal to $v=174 \mathrm{GeV}$. For heavy RH neutrinos $\left(M_{i} \gg v\right)$, light neutrino masses are obtained via the see-saw (type I) mechanism [5]

$$
m_{\nu}=U^{*} \hat{m}_{\nu} U^{\dagger}=\lambda_{\nu}^{T} \hat{M}_{\nu}^{-1} \lambda_{\nu} v^{2}
$$


where $U$ is the PMNS mixing matrix (and we adopt the convention of placing a hat over diagonal matrices with real and positive elements). In this framework the baryon asymmetry is generated by weak sphaleron processes converting the non-zero lepton number produced by outof-equilibrium decays of the heavy $\mathrm{RH}$ neutrinos.

The key quantities controlling the production of a net lepton number are the $\mathrm{CP}$ violating asymmetries in the $N_{i}$ decay rates

$$
\epsilon_{i \alpha} \equiv \frac{\Gamma\left(N_{i} \rightarrow \ell_{\alpha} \bar{H}\right)-\Gamma\left(N_{i} \rightarrow \bar{\ell}_{\alpha} H\right)}{\Gamma\left(N_{i} \rightarrow \ell_{\alpha} \bar{H}\right)+\Gamma\left(N_{i} \rightarrow \bar{\ell}_{\alpha} H\right)} .
$$

The inclusion of quantum effects discussed in the introduction (for technical details see Refs. [24, 26]) introduces a time dependence in the CP asymmetry

$$
\begin{aligned}
\epsilon_{i \alpha}(z) & =\sum_{j \neq i} \epsilon_{\alpha}^{(j, i)} m^{(i, j)}(z), \\
m^{(i, j)}(z) & =2 \sin ^{2}\left(\frac{1}{2} \frac{M_{j}-M_{i}}{2 H\left(M_{1}\right)} z^{2}\right)-\frac{\Gamma_{j}}{M_{j}-M_{i}} \sin \left(\frac{M_{j}-M_{i}}{2 H\left(M_{1}\right)} z^{2}\right), \\
\epsilon_{\alpha}^{(j, i)} & =\frac{1}{8 \pi} \frac{\operatorname{Im}\left[\left(\lambda_{\nu}\right)_{i \alpha}\left(\lambda_{\nu}\right)_{\alpha j}^{\dagger}\left(\lambda_{\nu} \lambda_{\nu}^{\dagger}\right)_{i j}\right]}{\left(\lambda_{\nu} \lambda_{\nu}^{\dagger}\right)_{i i}}\left(g_{s}^{(j, i)}+g_{v}^{(j, i)}\right), \\
g_{s}^{(j, i)} & =\sqrt{\frac{x_{j}}{x_{i}}} \frac{1}{1-\frac{x_{j}}{x_{i}}} \frac{1}{1+\frac{\Gamma_{j}^{2} / M_{i}^{2}}{\left(1-x_{j} / x_{i}\right)^{2}}}, \\
g_{v}^{(j, i)} & =\sqrt{\frac{x_{j}}{x_{i}}}\left(1-\left(1+\frac{x_{j}}{x_{i}}\right) \ln \frac{1+x_{j} / x_{i}}{x_{j} / x_{i}}\right),
\end{aligned}
$$

where $\Gamma_{j} \equiv \sum_{\beta} \Gamma\left(N_{j} \rightarrow \ell_{\beta} \bar{H}\right)=\left(\lambda_{\nu} \lambda_{\nu}^{\dagger}\right)_{j j} M_{j} /(8 \pi)$ is the total decay rate of the $j$-th RH neutrino, $H$ is the Hubble expansion rate, $z=M_{1} / T, T$ denotes the temperature, $g_{s}$ and $g_{v}$ are the selfenergy and the vertex correction functions respectively and $x_{i}=\left(M_{i} / M_{1}\right)^{2}$ (a short summary of the Boltzmann equations can be found in the Appendix A). The combination of Yukawa couplings appearing in Eq. (4) is quite constrained under the hypothesis of MLFV and, as we discuss below, the requirement of non-vanishing $\epsilon_{i \alpha}$ leads to non-trivial constraints on the free parameters of this framework.

In the quantum Boltzmann approach, the typical time-scale for the variation of the CP asymmetry is

$$
t=\frac{1}{2 H(T)}=\frac{z^{2}}{2 H\left(M_{1}\right)} \sim \frac{1}{M_{j}-M_{i}}=\frac{1}{\Delta M_{j i}} .
$$

As a consequence, quantum effects are expected to be sizable if $1 / \Delta M_{j i}$ is larger than the timescale for changing the abundance, $1 / \Gamma_{i}$. In other words, the oscillation frequency $\Delta M_{j i}$ has to be sufficiently smaller than $\Gamma_{i}$, so that the factors $m^{(i, j)}(z)$ do not effectively average to one. Under these conditions, the amplitude of the "sin" term in $m^{(i, j)}(z)$ is also enhanced, which will turn out to be a crucial effect. If $1 / \Delta M_{j i}$ is smaller than the time-scale for changing the particle abundances, then the CP asymmetry may be averaged over many scatterings [24] and it reduces to the classical value $\epsilon_{i \alpha}=\sum_{j \neq i} \epsilon_{\alpha}^{(j, i)}$.

The above discussion leads us to formulate a quantitative criterion for the importance of quantum effects, namely $\Delta M_{j i} \lesssim \Gamma_{i}$. This criterion can be naturally satisfied if RH neutrinos are 
nearly degenerate, as first pointed out in [26] and therefore in models based on MLFV. So the next task is to identify the parameters controlling the ratio $\Delta M_{j i} / \Gamma_{i}$ within the MLFV framework.

\section{The MLFV scenario}

The MLFV approach with extended field content [17] is based on the assumption that the largest possible flavour symmetry group of the lepton sector, $S U(3)_{\ell} \times S U(3)_{e} \times O(3)_{N}$, is broken only by two irreducible sources: the Yukawa couplings $\lambda_{e}^{0}$ and $\lambda_{\nu}^{0}$ transforming as $(\overline{3}, 3,1)$ and $(\overline{3}, 1,3)$ respectively.

In the limit of vanishing Yukawa couplings, the $O(3)_{N}$ symmetry is exact and RH neutrinos are degenerate at the common scale $M_{\nu}^{0}$. Switching on the Yukawa interactions $\lambda_{\nu}^{0}$ and $\lambda_{e}^{0}$, the mass degeneracy is removed as an effect of those combinations of Yukawa couplings transforming as $\left(1,1,6_{s}\right)[21$ :

$$
\begin{aligned}
M_{\nu}=M_{\nu}^{0}[1 & +c^{(1)}\left(h_{\nu}^{0}+h_{\nu}^{0^{T}}\right)+c_{1}^{(2)}\left(\left(h_{\nu}^{0}\right)^{2}+\left(h_{\nu}^{0^{T}}\right)^{2}\right) \\
& \left.+c_{2}^{(2)} h_{\nu}^{0} h_{\nu}^{0^{T}}+c_{3}^{(2)} h_{\nu}^{0^{T}} h_{\nu}^{0}+c_{4}^{(2)}\left(h_{e}^{0}+h_{e}^{0^{T}}\right)+\ldots\right],
\end{aligned}
$$

where $h_{\nu}^{0}=\lambda_{\nu}^{0} \lambda_{\nu}^{0^{\dagger}}, h_{e}^{0}=\lambda_{\nu}^{0} \lambda_{e}^{0^{\dagger}} \lambda_{e}^{0} \lambda_{\nu}^{0^{\dagger}}$ and one can choose $e^{1} \lambda_{e}^{0}=\hat{m}_{e} / v$. The coefficients $c$ 's are arbitrary parameters smaller than $\mathcal{O}(1)$. If one interprets them as arising from radiative effects, it is natural to have $c^{(1)} \sim 1 /(4 \pi)^{2}$ and $c_{i}^{(2)} \sim\left(c^{(1)}\right)^{2}$. From the unitary matrix $\bar{U}$ diagonalising $M_{\nu}$, one obtains $\hat{M}_{\nu}$ and $\lambda_{\nu}$ of Eqs. $(1)$ and (2):

$$
\hat{M}_{\nu}=\bar{U} M_{\nu} \bar{U}^{T} \quad, \quad \lambda_{\nu}=\bar{U} \lambda_{\nu}^{0} .
$$

Notice that, due to the smallness of the $c$ 's, it is natural to expect $\bar{U}$ to be close to the unity matrix (or possibly a permutation matrix, given the ordering convention $M_{1} \leq M_{2} \leq M_{3}$ ).

One can see $\lambda_{\nu}^{0}$ as the neutrino Yukawa coupling associated to degenerate RH neutrinos. Accordingly, a convenient parameterisation to be exploited in the following is 31]

$$
\lambda_{\nu}^{0}=\frac{1}{v} \sqrt{M_{\nu}^{0}} H \sqrt{\hat{m}_{\nu}^{0}} U^{0^{\dagger}}
$$

where $\hat{m}_{\nu}^{0}=\operatorname{diag}\left(m_{1}^{0}, m_{2}^{0}, m_{3}^{0}\right), U^{0}=R_{23}\left(\theta_{23}^{0}\right) \Gamma_{\delta^{0}} R_{13}\left(\theta_{13}^{0}\right) \Gamma_{\delta^{0}}^{\dagger} R_{12}\left(\theta_{12}^{0}\right) \operatorname{diag}\left(\mathrm{e}^{\mathrm{i} \frac{\alpha_{1}^{0}}{2}}, \mathrm{e}^{\mathrm{i} \frac{\alpha_{2}^{0}}{2}}, 1\right), \Gamma_{\delta^{0}}=$ $\operatorname{diag}\left(1,1, e^{i \delta^{0}}\right)$ and $H$ is an orthogonal hermitian matrix

$$
H=e^{i \Phi} \quad, \quad \Phi=\left(\begin{array}{ccc}
0 & \phi_{1} & \phi_{2} \\
-\phi_{1} & 0 & \phi_{3} \\
-\phi_{2} & -\phi_{3} & 0
\end{array}\right) \quad .
$$

Clearly, the smaller the splittings among the RH neutrinos, the closer $\hat{m}_{\nu}$ and $U$ are to $\hat{m}_{\nu}^{0}$ and $U^{0}$ respectively. Since the approximations $\hat{m}_{\nu} \approx \hat{m}_{\nu}^{0}$ and $U \approx U^{0}$ will turn out to be good, in

\footnotetext{
${ }^{1}$ The charged lepton and Dirac-neutrino Yukawa couplings themselves receive small corrections induced by $\lambda_{\nu, e}^{0}$; the effect of going in the basis where charged leptons are diagonal is accounted for by a redefinition of the $c$ 's in Eq. (6).
} 
all the subsequent discussion we drop the 0 -superscript above the parameters of $\hat{m}_{\nu}^{0}$ and $U^{0}$. For later convenience, notice also that

$$
\begin{aligned}
h_{\nu}^{0} & =\lambda_{\nu}^{0} \lambda_{\nu}^{0^{\dagger}}=H \hat{m}_{\nu}^{0} H \frac{M_{\nu}^{0}}{v^{2}}, \\
h_{e}^{0} & =\lambda_{\nu}^{0} \lambda_{e}^{0^{\dagger}} \lambda_{e}^{0} \lambda_{\nu}^{0^{\dagger}}=H E H \frac{M_{\nu}^{0}}{v^{2}} \quad, \quad E=\sqrt{\hat{m}_{\nu}^{0}} U^{0^{\dagger}}\left(\frac{\hat{m}_{e}}{v}\right)^{2} U^{0} \sqrt{\hat{m}_{\nu}^{0}} .
\end{aligned}
$$

\section{General Implications of MLFV for thermal leptogenesis}

The MLFV scenario described above has six independent CP-violating phases. As shown in Appendix B they can be characterized in terms of weak-basis invariants that in turn are in direct correspondence with linearly independent combinations of the asymmetries $\epsilon_{i \alpha}$. The analysis of weak-basis invariants (see Appendix B for more details) allows us to draw the following general conclusions on the viability of leptogenesis both in the one-flavour regime $\left(M_{\nu}^{0}>10^{12} \mathrm{GeV}\right)$ as well as in the flavour regime $\left(M_{\nu}^{0}<10^{12} \mathrm{GeV}\right)$ :

- In the one-flavour regime, in order to produce the lepton asymmetry one needs non-zero second-order RH mass splitting (i.e. $c_{i}^{(2)} \neq 0$ ) and $H \neq I$, i.e. $\mathrm{CP}$ violation (CPV) in the RH sector [21].

- Flavour effects open at least in principle two new regimes for MLFV-leptogenesis which are not allowed in the one-flavour limit: (i) the case in which RH mass splitting is induced only (or mainly) by $c^{(1)}$. This situation requires $H \neq I$, namely CPV in the RH sector; (ii) the case in which CPV arises only from PMNS phases [23], namely $H=I$ and $M_{\nu}$ is real. In this case the lepton asymmetry is proportional to $c_{4}^{(2)}$.

We first study the Yukawa-induced RH mass splitting. The largest correction term is naturally expected to be the one associated to $c^{(1)}$ in Eq. (6), so that many considerations about the $\mathrm{RH}$ neutrino spectrum and the frequency of the oscillations in $m^{(i, j)}$ can already be drawn. This program is straightforward in the limit of small $\phi_{i}$, which from the numerical analysis will turn out to be the relevant one in order to achieve viable leptogenesis. In this regime one can then expand the relevant matrices in series of $\phi_{i}$. In particular, one has from Eq. (8)

$$
\lambda_{\nu}^{0}=\frac{\sqrt{M_{\nu}^{0}}}{v}\left(\begin{array}{ccc}
e^{-i \frac{\alpha_{1}}{2}} \sqrt{m_{1}} c_{12} & -e^{-i \frac{\alpha_{1}}{2}} \sqrt{m_{1}} c_{23} s_{12} & e^{-i \frac{\alpha_{1}}{2}} \sqrt{m_{1}} s_{12} s_{23} \\
e^{-i \frac{\alpha_{2}}{2}} \sqrt{m_{2}} s_{12} & e^{-i \frac{\alpha_{2}}{2}} \sqrt{m_{2}} c_{12} c_{23} & -e^{-i \frac{\alpha_{2}}{2}} \sqrt{m_{2}} c_{12} s_{23} \\
\sqrt{m_{3}} s_{13} e^{i \delta} & \sqrt{m_{3}} s_{23} & \sqrt{m_{3}} c_{23}
\end{array}\right)+\mathcal{O}\left(\phi_{i}\right),
$$

where the 0-superscript is understood for $m_{i}$ and the parameters of $U^{0}$. In addition

$$
h_{\nu}^{0}=\frac{M_{\nu}^{0}}{v^{2}}\left(\begin{array}{ccc}
m_{1} & i \phi_{1}\left(m_{1}+m_{2}\right) & i \phi_{2}\left(m_{1}+m_{3}\right) \\
-i \phi_{1}\left(m_{1}+m_{2}\right) & m_{2} & i \phi_{3}\left(m_{2}+m_{3}\right) \\
-i \phi_{2}\left(m_{1}+m_{3}\right) & -i \phi_{3}\left(m_{2}+m_{3}\right) & m_{3}
\end{array}\right)+\mathcal{O}\left(\phi_{i} \phi_{j}\right)
$$

and

$h_{\nu}^{0}+h_{\nu}^{0^{T}}=\frac{M_{\nu}^{0}}{v^{2}} \times$ 


$$
\begin{aligned}
& \times\left(\begin{array}{ccc}
2 m_{1} & \phi_{2} \phi_{3}\left(m_{1}+m_{2}+2 m_{3}\right) & -\phi_{1} \phi_{3}\left(m_{1}+2 m_{2}+m_{3}\right) \\
\phi_{2} \phi_{3}\left(m_{1}+m_{2}+2 m_{3}\right) & 2 m_{2} & \phi_{1} \phi_{2}\left(2 m_{1}+m_{2}+m_{3}\right) \\
-\phi_{1} \phi_{3}\left(m_{1}+2 m_{2}+m_{3}\right) & \phi_{1} \phi_{2}\left(2 m_{1}+m_{2}+m_{3}\right) & 2 m_{3}
\end{array}\right) \\
& +\mathcal{O}\left(\phi_{i}^{3}\right) .
\end{aligned}
$$

The leading correction for RH neutrino masses is simply linked to the light neutrino mass spectrum

$$
M_{\nu}=M_{\nu}^{0}\left[1+2 c^{(1)} \frac{M_{\nu}^{0} \hat{m}_{\nu}^{0}}{v^{2}}\left(1+\mathcal{O}\left(\phi_{i} \phi_{j}\right)\right)\right] .
$$

Let us consider NH and IH for light neutrinos in turn. From the form of $M_{\nu}$ it turns out that in first approximation,

$$
\bar{U}^{N H} \approx I \quad, \quad \bar{U}^{I H} \approx\left(\begin{array}{ccc}
0 & 0 & 1 \\
1 & 0 & 0 \\
0 & 1 & 0
\end{array}\right)=\tilde{I} .
$$

Aiming at a compact notation, we introduce the matrix $\mathbf{m}=\operatorname{diag}\left(m_{l}, m_{i}, m_{h}\right)$, where $m_{l}<m_{i}<$ $m_{h}$ (light, intermediate, heavy); clearly, for NH $l=1, i=2, h=3$ while for $\mathrm{IH} l=3, i=1, h=2$. Then, defining $\Delta \mathbf{m}_{j i}=\mathbf{m}_{j}-\mathbf{m}_{i}$ one has from Eq.(15):

$$
M_{i} \approx M_{\nu}^{0}\left(1+2 c^{(1)} \frac{M_{\nu}^{0} \mathbf{m}_{i}}{v^{2}}\right) \quad, \quad \Delta M_{j i} \approx 2 c^{(1)} \Delta \mathbf{m}_{j i}\left(\frac{M_{\nu}^{0}}{v}\right)^{2},
$$

So we have identified the parameters that control the frequency of the oscillating $m^{(i, j)}$, see Eq. (4). Moreover, the RH neutrino widths and the amplitude $A_{j i}$ of the sin-term in $m^{(i, j)}$ can then be expressed as

$$
\Gamma_{j} \approx \frac{\mathbf{m}_{j}}{8 \pi}\left(\frac{M_{\nu}^{0}}{v}\right)^{2} \quad, \quad A_{j i} \equiv \frac{\Gamma_{j}}{\Delta M_{j i}} \approx \frac{1}{16 \pi c^{(1)}} \frac{\mathbf{m}_{j}}{\Delta \mathbf{m}_{j i}} .
$$

We are now in a position to study the condition for the importance of quantum effects in the CP asymmetries, namely $\Delta M_{j i}<\Gamma_{i}$. Using the expressions above, in the MLFV framework this condition can be cast into

$$
16 \pi c^{(1)} \frac{\Delta \mathbf{m}_{j i}}{\mathbf{m}_{i}}<1,
$$

implying that the difference between the classical and quantum approach will increase as $c^{(1)}$ becomes smaller and/or the pair $\mathbf{m}_{j}-\mathbf{m}_{i}$ becomes degenerate. In the next sections we will study numerically and analytically the dependence of the baryon asymmetry on $c^{(1)}$ and the lightest neutrino mass $m_{l}$, controlling the degeneracy of the light neutrino spectrum.

Both in the classical and quantum case, in order for $\epsilon_{\alpha}^{(j, i)}$ to be non vanishing, it is crucial to have non diagonal entries in $h_{\nu}=\lambda_{\nu} \lambda_{\nu}^{\dagger}=\bar{U} h_{\nu}^{0} \bar{U}^{\dagger}$. Since $\bar{U}$ is a small rotation, one has $h_{\nu} \sim h_{\nu}^{0}$. From Eq. (13) it turns out that the simplest situation in which $h_{\nu}$ is non-diagonal occurs when at least one among the $\phi_{i}$ is non vanishing, namely $H \neq 1$. On the other hand, in the case $H=1$, small real non diagonal elements for $h_{\nu}$ have to be generated as an effect of $\bar{U}$; in this case the CP violation needed for a non-zero asymmetry arises from the PMNS phases in $\left(\lambda_{\nu}\right)_{i \alpha}$. These two situations are going to be discussed separately in the next sections.

Before turning to analyse the differences between the quantum and classical approaches focussing on these two relevant cases, we show the expressions for the washout parameters. For 
both $\mathrm{NH}$ and IH, the washout parameters $K_{\alpha}=\sum_{i} K_{i \alpha}$ and $K_{i}=\sum_{\alpha} K_{i \alpha}$ (see appendix A for more details) are given by:

$$
\begin{aligned}
K_{e} & =\left(m_{1} c_{12}^{2}+m_{2} s_{12}^{2}+m_{3} s_{13}^{2}\right) / m_{*}, \\
K_{\mu} & =\left(m_{1} c_{23}^{2} s_{12}^{2}+m_{2} c_{12}^{2} c_{23}^{2}+m_{3} s_{23}^{2}\right) / m_{*}, \\
K_{\tau} & =\left(m_{1} s_{12}^{2} s_{23}^{2}+m_{2} c_{12}^{2} s_{23}^{2}+m_{3} c_{23}^{2}\right) / m_{*}, \\
K_{i} & =\mathbf{m}_{i} / m_{*} \quad(i=1,2,3) .
\end{aligned}
$$

It is clear that none of the $K_{\alpha}(\alpha=e, \mu, \tau)$ can be small: the weak-washout regime cannot be obtained in the framework of MLFV. However, let us emphasise that for NH the potentially smallest washout parameter is $K_{e}$. On the contrary, for IH all the $K_{\alpha}$ are comparable and relatively large (in particular, for small $m_{3}$ one has the relation $K_{e} \sim m_{1} / m_{*} \sim 2 K_{\mu, \tau}$ ).

In what follows, we shall focus our attention on the full flavoured regimf ${ }^{2}, M_{\nu}^{0} \lesssim 10^{9} \mathrm{GeV}$, and we shall illustrate the impact of quantum effects in the two cases in which $\mathrm{CP}$ asymmetries arise either from the RH $(H \neq I)$ sector or from the PMNS phases $(H=I)$.

\section{$5 \quad$ CP Violation from $H \neq I$}

We first analyse the situation $c^{(1)} \gg c_{i}^{(2)}$ and $H \neq I$, in which the lepton asymmetries are proportional to the angles $\phi_{i}$. For NH, let us focus on the asymmetries associated to the least washed-out flavour, that is the $e$-flavour. Introducing the notation $F^{(j, i)}=1 /(8 \pi)\left(g_{s}^{(j, i)}+g_{v}^{(j, i)}\right) m^{(i, j)}$ and by working at first order in $\phi_{i}$, we find:

$$
\begin{gathered}
\epsilon_{1 e}=\sqrt{\frac{m_{2}}{m_{1}}} \frac{m_{1}+m_{2}}{\tilde{m}} c_{12} s_{12} \phi_{1} F^{(2,1)} \cos \left(\frac{\alpha_{2}-\alpha_{1}}{2}\right)+\sqrt{\frac{m_{3}}{m_{1}}} \frac{m_{1}+m_{3}}{\tilde{m}} c_{12} s_{13} \phi_{2} F^{(3,1)} \cos \left(\frac{2 \delta+\alpha_{1}}{2}\right), \\
\epsilon_{2 e}=-\sqrt{\frac{m_{1}}{m_{2}}} \frac{m_{1}+m_{2}}{\tilde{m}} c_{12} s_{12} \phi_{1} F^{(1,2)} \cos \left(\frac{\alpha_{2}-\alpha_{1}}{2}\right)+\sqrt{\frac{m_{3}}{m_{2}}} \frac{m_{2}+m_{3}}{\tilde{m}} s_{12} s_{13} \phi_{3} F^{(3,2)} \cos \left(\frac{2 \delta+\alpha_{2}}{2}\right), \\
\epsilon_{3 e}=-\sqrt{\frac{m_{1}}{m_{3}}} \frac{m_{1}+m_{3}}{\tilde{m}} c_{12} s_{13} \phi_{2} F^{(1,3)} \cos \left(\frac{2 \delta+\alpha_{1}}{2}\right)-\sqrt{\frac{m_{2}}{m_{3}}} \frac{m_{2}+m_{3}}{\tilde{m}} s_{12} s_{13} \phi_{3} F^{(2,3)} \cos \left(\frac{2 \delta+\alpha_{2}}{2}\right),
\end{gathered}
$$

where $\tilde{m}=v^{2} / M_{\nu}^{0}$. For zero CPV in PMNS the $\epsilon_{i \alpha}$ are maximised. Notice that for some values of the PMNS phases, one can suppress $\eta_{B}$; however, if all $\phi_{i} \neq 0$, there is no choice of PMNS phases that allows all three $\epsilon$ to vanish ${ }^{3}$.

\subsection{Dependences on $c^{(1)}$ and $m_{l}$}

In order to explore numerically the dependence on $c^{(1)}$, we set $c_{i}^{(2)}=0$ and choose $M_{\nu}^{0}=10^{9}$ $\mathrm{GeV}$. Then, we switch on in turn each $\phi_{i}=10^{-4}$, setting to zero the two others. Because of the smallness of the $\phi_{i}$, we will have $\hat{m}_{\nu} \approx \hat{m}_{\nu}^{0}$ and $U \approx U^{0}$; for definiteness, we select the parameters of $U^{0}$ to be: $\theta_{23}=\pi / 4, \theta_{12}=35^{\circ}, \theta_{13}=10^{-3}, \delta=\alpha_{1}=\alpha_{2}=0$.

For $m_{l}=10^{-3} \mathrm{eV}$ (thick red line) and $m_{l}=10^{-1} \mathrm{eV}$ (thin blue line), the upper plots of Figs. 1 . and 2 show the ratio of the asymmetry computed including quantum effects $\left(\eta_{B}^{\mathrm{qu}}\right)$ to the classical

\footnotetext{
${ }^{2}$ For $M_{\nu}^{0}>10^{9} \mathrm{GeV}$ the results do not change significantly.

${ }^{3}$ Indeed, the term proportional to $\left\{\phi_{1}, \phi_{2}, \phi_{3}\right\}$ vanishes when $\left\{\alpha_{2}-\alpha_{1}, 2 \delta+\alpha_{1}, 2 \delta+\alpha_{2}\right\}=\pi+\bmod (2 \pi)$, respectively. The latter two conditions imply $\alpha_{2}-\alpha_{1}=0+\bmod (2 \pi)$, in conflict with the first condition.
} 

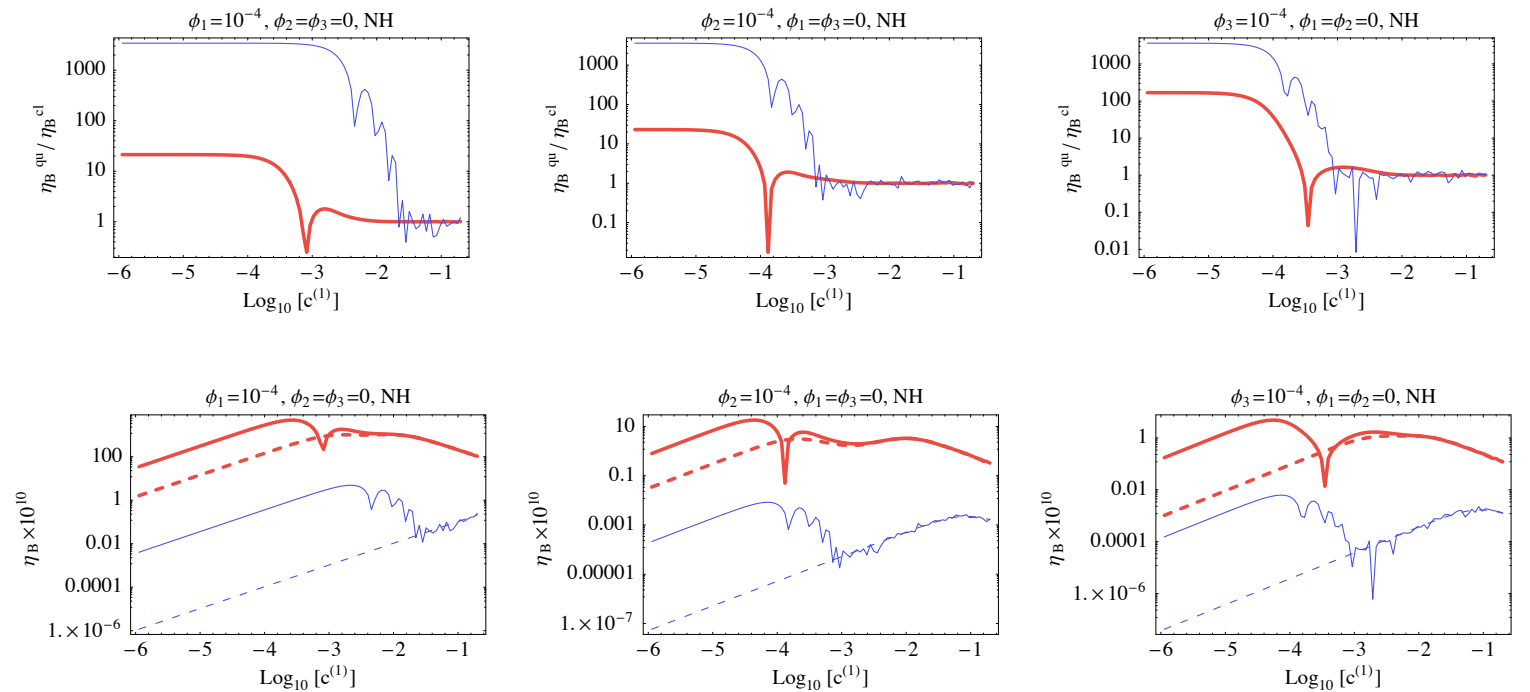

Figure 1: Dependence on $c^{(1)}$, in normal hierarchy. Thick red and thin blue lines correspond to $m_{1}=10^{-3} \mathrm{eV}$ and $m_{1}=10^{-1} \mathrm{eV}$, respectively. Top: The absolute value of the ratio of the baryon asymmetry with quantum effects $\left(\eta_{B}^{\mathrm{qu}}\right)$ and without quantum effects $\left(\eta_{B}^{\mathrm{cl}}\right)$. Bottom: The absolute values of $\eta_{B}^{\mathrm{qu}}$ (solid lines) and $\eta_{B}^{\mathrm{cl}}$ (dashed lines).
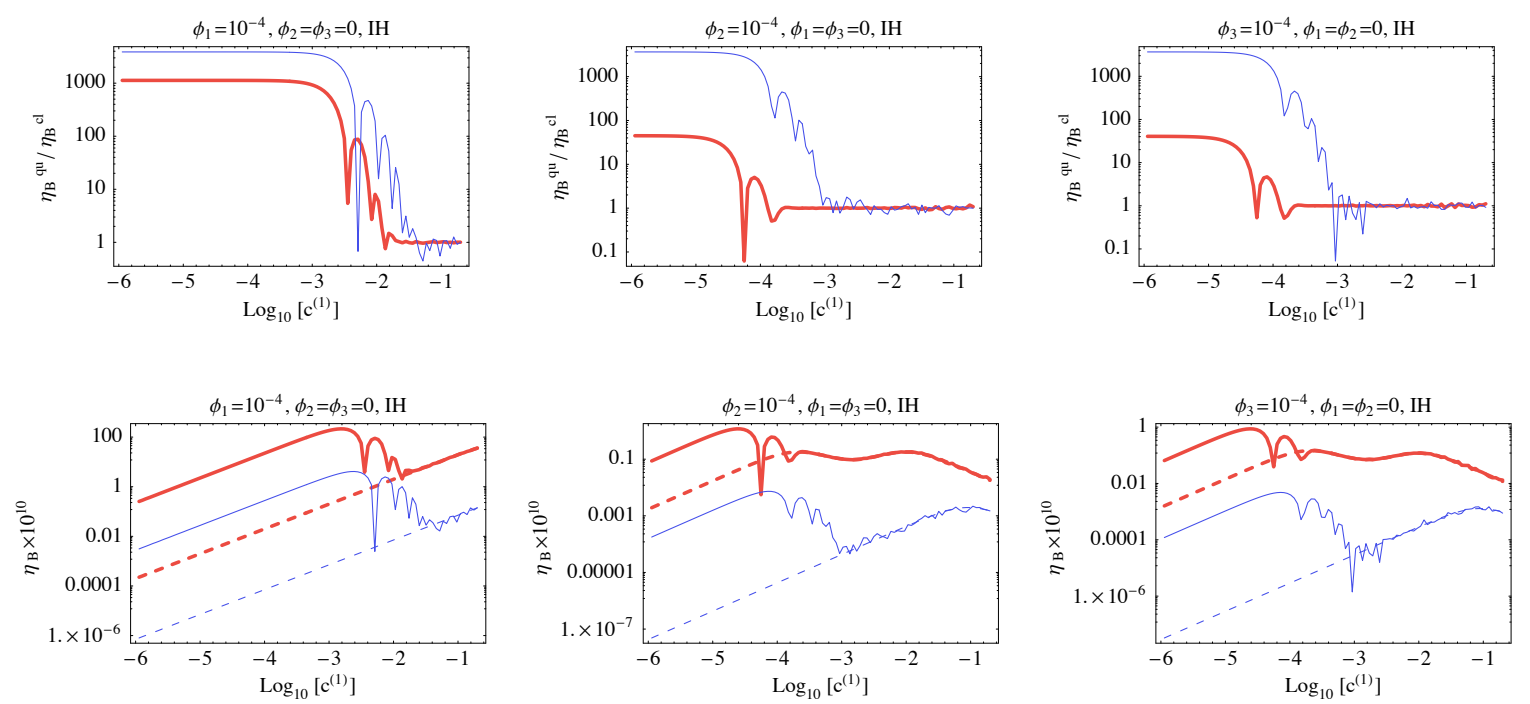

Figure 2: Dependence on $c^{(1)}$, in inverse hierarchy. Thick red and thin blue lines correspond to $m_{1}=10^{-3} \mathrm{eV}$ and $m_{1}=10^{-1} \mathrm{eV}$, respectively. Top: The absolute value of the ratio of the baryon asymmetry with quantum effects $\left(\eta_{B}^{\mathrm{qu}}\right)$ and without quantum effects $\left(\eta_{B}^{\mathrm{cl}}\right)$. Bottom: The absolute values of $\eta_{B}^{\mathrm{qu}}$ (solid lines) and $\eta_{B}^{\mathrm{cl}}$ (dashed lines). 

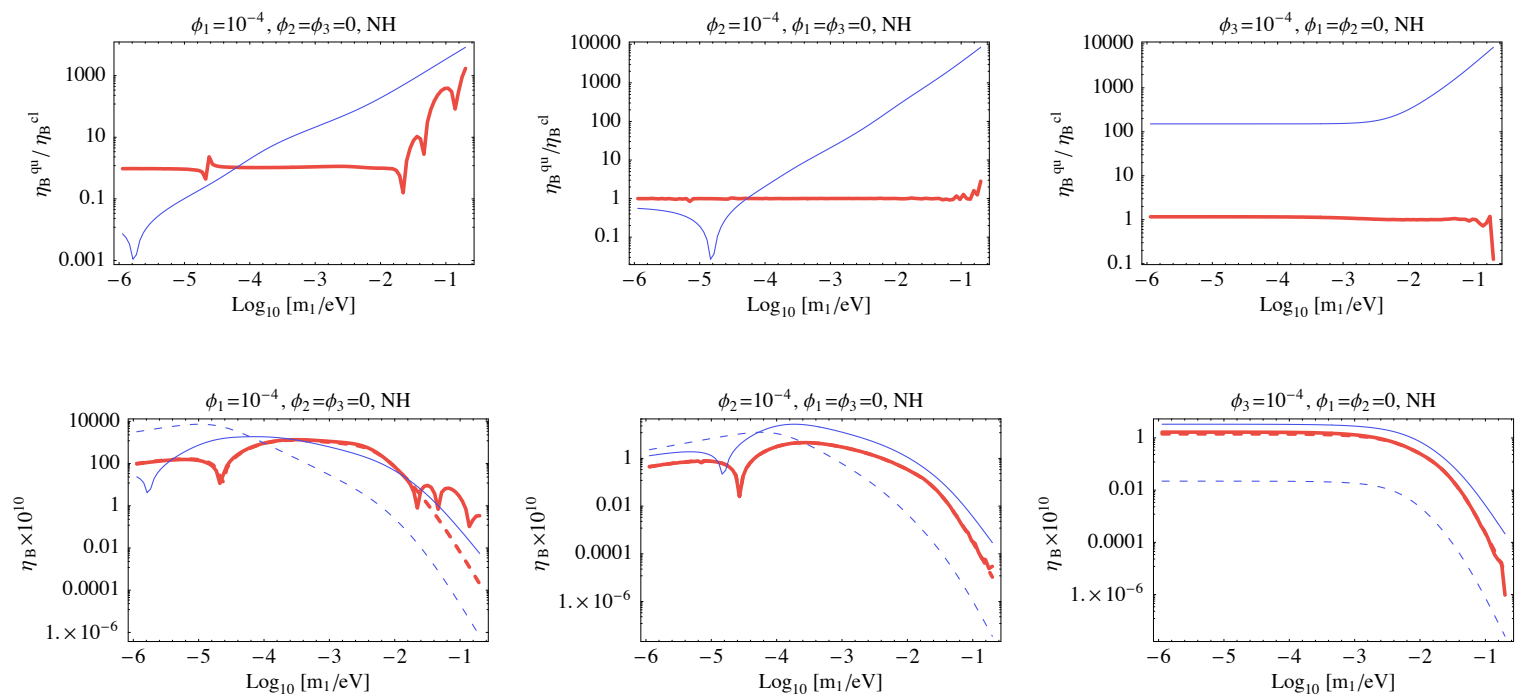

Figure 3: Dependence on $m_{1}$, in normal hierarchy. Thick red and thin blue lines correspond to $c^{(1)}=6 \times 10^{-3}$ and $c^{(1)}=2 \times 10^{-5}$, respectively. Top: The absolute value of the ratio of the baryon asymmetry with quantum effects $\left(\eta_{B}^{\mathrm{qu}}\right)$ and without quantum effects $\left(\eta_{B}^{\mathrm{cl}}\right)$. Bottom: The absolute values of $\eta_{B}^{\mathrm{qu}}$ (solid lines) and $\eta_{B}^{\mathrm{cl}}$ (dashed lines).
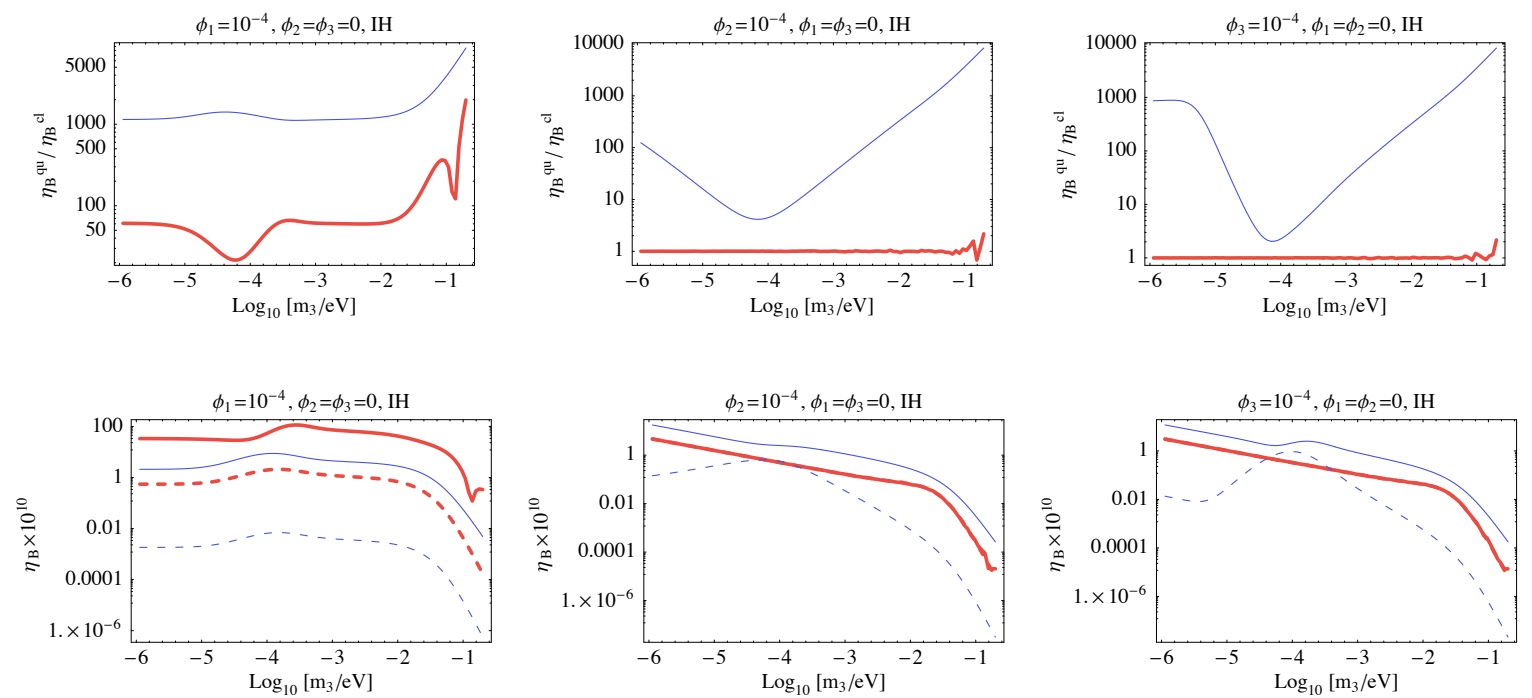

Figure 4: Dependence on $m_{3}$, in inverse hierarchy. Thick red and thin blue lines correspond to $c^{(1)}=6 \times 10^{-3}$ and $c^{(1)}=2 \times 10^{-5}$, respectively. Top: The absolute value of the ratio of the baryon asymmetry with quantum effects $\left(\eta_{B}^{\mathrm{qu}}\right)$ and without quantum effects $\left(\eta_{B}^{\mathrm{cl}}\right)$. Bottom: The absolute values of $\eta_{B}^{\mathrm{qu}}$ (solid lines) and $\eta_{B}^{\mathrm{cl}}$ (dashed lines). 
asymmetry $\left(\eta_{B}^{\mathrm{cl}}\right)$, for $\mathrm{NH}$ and $\mathrm{IH}$ respectively. The corresponding lower plots show the absolute value of $\eta_{B}$ with (solid line) and without (dashed line) quantum effects. In the classical case, the resonant point is usually reached for $c^{(1)} \sim 0.01$, the only exception being IH with $\phi_{1} \neq 0$ where it is reached for $c^{(1)}=\mathcal{O}(1)$. Let us recall that the natural range for radiatively-induced $c^{(1)}$ is around $1 /(4 \pi)^{2}=6 \times 10^{-3}$. As can be seen, quantum effects induce an enhancement of $\eta_{B}$ at small values of $c^{(1)}$. Such an enhancement is caused mainly by the second $(\sin )$ term of the function $m^{(i, j)}$. For both NH and IH quantum effects seem to be important for $c^{(1)} \lesssim 10^{-3}$, the only exception being the case of nearly degenerate light neutrinos with $\phi_{1}$ as the main source of CPV in leptogenesis. In the latter case, values of $c^{(1)}$ up to $10^{-2}$ could do the job.

The figures also show that with degenerate light neutrinos quantum effects are larger, as expected, but $\eta_{B}$ is more suppressed. Since the baryon asymmetry scales linearly with $\phi_{i}$ one would need these phases to be larger than about $10^{-4}$ to reproduce the observed value of the baryon asymmetry. The dependence on $m_{l}$ is shown in more detail by the plots of Figs. 3 and 4 for $\mathrm{NH}$ and IH respectively, selecting two representative values of $c^{(1)}: c^{(1)}=6 \times 10^{-3}$ (radiatively induced) and $c^{(1)}=2 \times 10^{-5}$. Notice that the inclusion of the terms proportional to $c_{1}^{(2)}$, setting for instance $c_{1}^{(2)}=\left(c^{(1)}\right)^{2}$ in Eq. (6), does not change the above results.

\subsection{Analytical estimates}

In this subsection we will derive a simple analytical estimate for the ratio

$$
\left.R \equiv \frac{\left|\eta_{B}^{\mathrm{qu}}\right|}{\left|\eta_{B}^{\mathrm{cl}}\right|}\right|_{c^{(1)} \rightarrow 0}=\left.\frac{\left|\sum_{\alpha}\left(Y_{\Delta_{\alpha}}\right)^{\mathrm{qu}}\right|}{\left|\sum_{\alpha}\left(Y_{\Delta_{\alpha}}\right)^{\mathrm{cl}}\right|}\right|_{c^{(1)} \rightarrow 0}
$$

and check that it is in agreement with the numerical findings.

The dominant contribution to the asymmetry will come from the least washed-out flavour $\beta$, e.g. the one with the smallest $K_{\beta}$. Therefore, as a first approximation we take:

$$
\left.R \simeq \frac{\left|\left(Y_{\Delta_{\beta}}\right)^{\mathrm{qu}}\right|}{\left|\left(Y_{\Delta_{\beta}}\right)^{\mathrm{cl}}\right|}\right|_{C^{(1) \rightarrow 0}} .
$$

In general, $Y_{\Delta_{\beta}}$ receives three contributions from the out-of-equilibrium decays of the three $N_{i}$ 's. Each of these terms is proportional to the CP asymmetry $\epsilon_{i \beta}$, which contains $\left(\lambda_{\nu} \lambda_{\nu}^{\dagger}\right)_{i j} m^{(i, j)}$, with $j \neq i$. The expression 13 for $\lambda_{\nu} \lambda_{\nu}^{\dagger}$ tells us that if e.g. only $\phi_{1} \neq 0$, the only non-zero off-diagonal entries are those with $(i, j)=(1,2)$ and $(2,1)$.

In the limit $c^{(1)} \rightarrow 0$, the mass splittings $\Delta M_{j i}$ become small (compared to the scale $M_{\nu}^{0}$ ), and so the arguments of the periodic functions in $m^{(i, j)}$ are also small, in the range of $z$ where the lepton asymmetry is generated. The $\sin ^{2}$ term in $m^{(i, j)}$ is completely negligible; instead, the sin term has in front an amplitude proportional to $1 / \Delta M_{j i}$. Therefore, the dependence on $c^{(1)}$ cancels in this limit and the resulting quantity is

$$
m^{(i, j)}(z) \simeq-\frac{\Gamma_{j}}{2 H\left(M_{1}\right)} z^{2} \simeq-\frac{1}{2} K_{j} z^{2} .
$$

This disappearance of $c^{(1)}$ explains the plateaux observed in the upper plots of Figs. 1 and 2 in the limit of very small $c^{(1)}$. 
Let us consider the case where only $\phi_{i} \neq 0$. Looking at the expression $(13)$, we only have two non-zero asymmetries, say $\epsilon_{j \beta}$ and $\epsilon_{k \beta}$, but we choose the one containing the least washout parameter $K_{j}$, since it corresponds to the most out-of-equilibrium RH neutrino whose decay produces the largest asymmetry; so we just take $\epsilon_{j \beta}(z) \simeq-\bar{\epsilon}_{j \beta} K_{j} z^{2} / 2$, where $\bar{\epsilon}_{j \beta}$ is the $\mathrm{CP}$ asymmetry without the memory factor.

Our choices for the lightest neutrino masses $\left(m_{l}=10^{-3} \mathrm{eV}, 10^{-1} \mathrm{eV}\right)$ make all of the $K_{i}$ 's greater than or of order 1 . In such a case, it is a good approximation to take $\mathrm{d} Y_{N_{j}} / \mathrm{d} z \simeq \mathrm{d} Y_{N_{j}}^{\mathrm{eq}} / \mathrm{d} z=$ $-z^{2} \mathcal{K}_{1}(z) / 4 g_{*}$ (here and in the following $\mathcal{K}_{1}, \mathcal{K}_{2}$ are the modified Bessel functions of the first and second kind, respectively). Then, an approximate solution to the Boltzmann equations (Eqs. 61) and 62 in Appendix A is found using the steepest descent method:

$$
\begin{aligned}
\left(Y_{\Delta_{\beta}}\right)^{\mathrm{cl}} & \simeq-\frac{1}{4 g_{*}} \int_{0}^{\infty} \mathrm{d} z \bar{\epsilon}_{j \beta} z^{2} \mathcal{K}_{1}(z) e^{-\frac{K_{\beta}\left|A_{\beta \beta}\right|}{4} \int_{z}^{\infty} \mathrm{d} z^{\prime} z^{\prime 3} \mathcal{K}_{1}\left(z^{\prime}\right)} \\
& \simeq-\frac{\epsilon_{j \beta}^{-}}{g_{*}} \frac{1}{K_{\beta}\left|A_{\beta \beta}\right| \bar{z}_{1}}
\end{aligned}
$$

where $\bar{z}_{1}$ satisfies the condition: $\frac{K_{\beta}\left|A_{\beta \beta}\right|}{4} \bar{z}_{1}^{3} \mathcal{K}_{1}\left(\bar{z}_{1}\right)+\frac{3}{\bar{z}_{1}}-\frac{\mathcal{K}_{2}\left(\bar{z}_{1}\right)}{\mathcal{K}_{1}\left(\bar{z}_{1}\right)} \simeq 0$. A good analytical approximation for $\bar{z}_{1}$, valid for $K_{\beta} \gtrsim 1$, is $\bar{z}_{1} \simeq 3.47\left[\log \left(K_{\beta}\right)\right]^{0.64}$.

Taking into account the quantum correction factor (27) gives:

$$
\begin{aligned}
\left(Y_{\Delta_{\beta}}\right)^{\mathrm{qu}} & \simeq-\frac{1}{4 g_{*}} \int_{0}^{\infty} \mathrm{d} z \epsilon_{j \beta}(z) z^{2} \mathcal{K}_{1}(z) e^{-\frac{K_{\beta}\left|A_{\beta \beta}\right|}{4} \int_{z}^{\infty} \mathrm{d} z^{\prime} z^{\prime 3} \mathcal{K}_{1}\left(z^{\prime}\right)} \\
& \simeq \frac{\bar{\epsilon}_{j \beta}}{8 g_{*}} K_{j} \int_{0}^{\infty} \mathrm{d} z z^{4} \mathcal{K}_{1}(z) e^{-\frac{K_{\beta}\left|A_{\beta \beta}\right|}{4} \int_{z}^{\infty} \mathrm{d} z^{\prime} z^{\prime 3} \mathcal{K}_{1}\left(z^{\prime}\right)} \\
& \simeq \frac{\bar{\epsilon}_{j \beta}}{2 g_{*}} \frac{K_{j}}{K_{\beta}\left|A_{\beta \beta}\right|} \bar{z}_{2} .
\end{aligned}
$$

where $\bar{z}_{2}$ satisfies the condition: $\frac{K_{\beta}\left|A_{\beta \beta}\right|}{4} \bar{z}_{2}^{3} \mathcal{K}_{1}\left(\bar{z}_{2}\right)+\frac{4}{\bar{z}_{2}}-\frac{\mathcal{K}_{2}\left(\bar{z}_{2}\right)}{\mathcal{K}_{1}\left(\bar{z}_{2}\right)} \simeq 0$. Since one expects $\bar{z}_{1,2}>1$, they satisfy almost the same condition; thus it is reasonable to take: $\bar{z}_{1} \simeq \bar{z}_{2} \equiv \bar{z}$. The expressions (29) and (32) lead to the simple estimate:

$$
R\left(\phi_{i} \neq 0\right) \simeq \frac{1}{2} K_{j} \bar{z}^{2} \sim 10 K_{j}\left[\log \left(K_{\beta}\left|A_{\beta \beta}\right|\right)\right]^{1.28},
$$

where we neglected $\mathcal{O}(1)$ factors, since we are only interested in getting the order of magnitude of the importance of the quantum effects over the classical approximation. If we had taken lower values of $m_{l}$, thus giving $K_{j}=\min \left(K_{i}\right)<1$, the analytical estimate would have proceeded in a different way. Though, $R$ receives a leading contribution still proportional to $K_{j}$ as in (33), plus corrections going like $\frac{1}{z_{\mathrm{eq}}^{2}} \frac{K_{\beta}}{K_{j}}$, where $z_{\mathrm{eq}}$ is defined as the "time" at which the number density of $N_{j}$ reaches the equilibrium one: $Y_{N_{j}}\left(z_{\text {eq }}\right)=Y_{N_{j}}^{\mathrm{eq}}\left(z_{\text {eq }}\right)$. For the reference values we considered, this additional term is $\sim 10^{-2} K_{\beta} / K_{j}$. As described later, such a correction might be important in the limit of very small $K_{j}$, i.e. very small $m_{l}$, since the leading term is suppressed in that limit.

Let us now apply the analytical result (33) to the specific cases analysed numerically in the previous subsection.

Normal Hierarchy. With the parameters chosen for the numerical analysis, the least washedout flavour is $\beta=e$, while the $K_{i}$ 's are in the order $K_{1}<K_{2}<K_{3}$. Thus, the previous arguments 
lead to

$$
R\left(\phi_{1} \neq 0\right) \simeq R\left(\phi_{2} \neq 0\right) \sim 10 K_{1}\left[\log \left(K_{e}\left|A_{e e}\right|\right)\right]^{1.28}, \quad R\left(\phi_{3} \neq 0\right) \sim 10 K_{2}\left[\log \left(K_{e}\left|A_{e e}\right|\right)\right]^{1.28} .
$$

In the case $m_{1}^{0}=10^{-3} \mathrm{eV}$, one has $K_{1} \simeq 0.9, K_{2} \simeq 8.4$ and $K_{e} \simeq 3.4$, giving

$$
R\left(\phi_{1} \neq 0\right) \simeq R\left(\phi_{2} \neq 0\right) \sim \mathcal{O}(10), \quad R\left(\phi_{3} \neq 0\right) \sim \mathcal{O}\left(10^{2}\right)
$$

In the case $m_{1}^{0}=10^{-1} \mathrm{eV}$, one has $K_{1} \simeq 93.5, K_{2} \simeq 93.8$ and $K_{e} \simeq 93.6$, giving

$$
R\left(\phi_{1} \neq 0\right) \simeq R\left(\phi_{2} \neq 0\right) \simeq R\left(\phi_{3} \neq 0\right) \sim \mathcal{O}\left(10^{4}\right) .
$$

Such estimates are in good agreement with the numerical values for $\eta_{B}^{\mathrm{qu}} / \eta_{B}^{\mathrm{cl}}$ in the $c^{(1)} \rightarrow 0$ limit, shown in the upper plots of Fig. 1 .

Inverse Hierarchy. In this case the least washed-out flavour is $\beta=\mu$, while the $K_{i}$ 's are in the order $K_{3}<K_{1}<K_{2}$. Repeating the same analysis as before, one finds

$$
R\left(\phi_{1} \neq 0\right) \sim 10 K_{1}\left[\log \left(K_{\mu}\left|A_{\mu \mu}\right|\right)\right]^{1.28}, \quad R\left(\phi_{2} \neq 0\right) \simeq R\left(\phi_{3} \neq 0\right) \sim 10 K_{3}\left[\log \left(K_{\mu}\left|A_{\mu \mu}\right|\right)\right]^{1.28} .
$$

In the case $m_{3}^{0}=10^{-3} \mathrm{eV}$, one has $K_{1} \simeq 46, K_{3} \simeq 0.9$ and $K_{\mu} \simeq 24$, giving

$$
R\left(\phi_{1} \neq 0\right) \sim \mathcal{O}\left(10^{3}\right), \quad R\left(\phi_{2} \neq 0\right) \simeq R\left(\phi_{3} \neq 0\right) \sim \mathcal{O}\left(10^{2}\right) .
$$

In the case $m_{3}^{0}=10^{-1} \mathrm{eV}$, one has $K_{1} \simeq 104, K_{2} \simeq 93$ and $K_{\mu} \simeq 10^{2}$, giving

$$
R\left(\phi_{1} \neq 0\right) \simeq R\left(\phi_{2} \neq 0\right) \simeq R\left(\phi_{3} \neq 0\right) \sim \mathcal{O}\left(10^{4}\right) .
$$

Again, these orders of magnitudes agree very well with the $c^{(1)} \rightarrow 0$ limit found by numerical integration, as one can see from the upper plots of Fig. 2.

As anticipated before, the same analytical approximation (33) qualitatively explains also part of the dependence on $m_{l}$, for very small $c^{(1)}$ (the thin blue line in the upper plots of Figs. 3 and 4 ). Indeed, by considering the various $K_{\alpha}$ and $K_{i}$ as functions of the light neutrino masses, as given by Eqs. (20)-(23), one can recover the decreasing behaviour of $R$ as $m_{l}$ becomes small. In some cases, though, the main term in $R$ given by (33) turns out to be proportional to $m_{l}$ itself. They are the cases $\phi_{1}, \phi_{2} \neq 0$ for $\mathrm{NH}$ and $\phi_{2}, \phi_{3} \neq 0$ for IH. In such situations, when $m_{l}$ is too small (compared to $m_{*} \simeq 10^{-3} \mathrm{eV}$ ) the leading contribution to $R$ is suppressed and the next-to-leading term proportional to $1 / K_{j}$ starts being important. This describes the change of behaviour which can be seen in the upper plots of Figs. 3 and 4 , in the cases we mentioned above.

The fact that such a change in $R$ occurs at different values of $m_{l}$ for $\mathrm{NH}$ and $\mathrm{IH}$ can be seen as follows. For NH, the next-to-leading order term contains $K_{e} / K_{1}$, as functions of the light neutrino masses; since $s_{13}$ is tiny, the main contribution will come from the term with $m_{2} / m_{1}=m_{i} / m_{l}$. On the contrary, for IH the largest term in the ratio $K_{\mu} / K_{3}$ is the one with $m_{1} / m_{3}=m_{h} / m_{l}$, which is generally an order of magnitude greater than $m_{i} / m_{l}$ for NH. Therefore, the next-to-leading term starts being relevant, in $\mathrm{IH}$, at a larger value of $m_{l}$ than in $\mathrm{NH}$.

In this Section we have considered the effect of the splitting induced by $c^{(1)}$ - namely $\phi_{i}$ as sources of CPV. We have found that what is important for quantum effects to be sizable is not the magnitude of the washout parameters $K$, which is independent of $c^{(1)}$ in the MLFV framework. Rather, what is crucial is the frequency and the amplitude of the sin oscillating term, which have to be respectively small and large. This requires small $c^{(1)}$ and/or strong degeneracy among the light neutrinos $\mathbf{m}_{j}$ and $\mathbf{m}_{i}$ for the quantum effects to be sizeable. In the next Section we will analyse the case in which $H=I$. 


\section{CP violation from light neutrino mixing $(H=I)$.}

MLFV-leptogenesis is viable in the limit $H=I$ only in the "flavoured" regime, provided that the heavy neutrino mass matrix receives a radiative splitting proportional to the charged-lepton Yukawa couplings $\left(\delta M_{\nu}=c_{4}^{(2)} M_{\nu}^{0}\left(h_{e}+h_{e}^{T}\right)\right)$. Here we derive the analytic dependence of the asymmetries $\epsilon_{i \alpha}$ on the parameters of the model.

The CP asymmetries read

$$
\epsilon_{i \alpha}=\sum_{j \neq i} F^{(j, i)} \frac{\operatorname{Im}\left(\left(\lambda_{\nu}\right)_{i \alpha}\left(\lambda_{\nu}^{\dagger}\right)_{\alpha j}\left(\lambda_{\nu} \lambda_{\nu}^{\dagger}\right)_{i j}\right)}{\left|\lambda_{\nu} \lambda_{\nu}^{\dagger}\right|_{i i}},
$$

where, following the notation of Section $3, \lambda_{\nu}=\bar{U} \lambda_{\nu}^{0}$ and $\bar{U} M_{\nu} \bar{U}^{T}=\hat{M}_{\nu}$. In the limit $\bar{U} \rightarrow 1$, where $\lambda \rightarrow \lambda_{\nu}^{0}$ (see Eq. (8)), it is easy to verify that the $\epsilon_{i \alpha}$ vanish. The key ingredient in obtaining a non-zero result is to have non-diagonal entries in $h_{\nu} \equiv \lambda_{\nu} \lambda_{\nu}^{\dagger}$ and correspondingly a non trivial $\bar{U}$ matrix. In order to estimate the off-diagonal entries of $h_{\nu}$ and $\bar{U}$ we perform a perturbative expansion of $M_{\nu}$ assuming $c_{i}^{(2)} \ll c^{(1)}$ :

$$
\begin{aligned}
M_{\nu} & =M_{\nu}^{0}\left\{I+2 c^{(1)} h_{\nu}^{0}+\left(2 c_{1}^{(2)}+c_{2}^{(2)}+c_{3}^{(2)}\right)\left(h_{\nu}^{0}\right)^{2}+c_{4}^{(2)}\left(h_{e}+h_{e}^{T}\right)+\cdots\right\}, \\
& =M+\Delta .
\end{aligned}
$$

Here $M$ is a real diagonal matrix, with eigenvalues $M_{i}=M_{\nu}^{0}\left[1+2 c^{(1)} \mathbf{m}_{i} / \tilde{m}+\left(2 c_{1}^{(2)}+c_{2}^{(2)}+\right.\right.$ $\left.\left.c_{3}^{(2)}\right)\left(\mathbf{m}_{i} / \tilde{m}\right)^{2}\right]$, and $\Delta$ is a real symmetric matrix given by

$$
\Delta= \begin{cases}c_{4}^{(2)} M_{\nu}^{0}\left(h_{e}+h_{e}^{T}\right) & \mathrm{NH} \\ c_{4}^{(2)} M_{\nu}^{0} \tilde{I}\left(h_{e}+h_{e}^{T}\right) \tilde{I}^{T} & \mathrm{IH}\end{cases}
$$

with the $\tilde{I}$ defined in Eq. (16). $M_{\nu}$ is diagonalised perturbatively by the real orthogonal matrix $\bar{U}=I+T\left(T^{T}=-T\right)$, with

$$
T_{i j}=\frac{\Delta_{i j}}{M_{i}-M_{j}}
$$

To first order in $c_{4}^{(2)} / c^{(1)}$ the explicit epression for $\lambda_{\nu} \lambda_{\nu}^{\dagger}=\bar{U} h_{\nu}^{0} \bar{U}^{\dagger}$ is then

$$
\left(\lambda_{\nu} \lambda_{\nu}^{\dagger}\right)_{i \neq j}=\frac{\Delta_{i j}\left(\mathbf{m}_{j}-\mathbf{m}_{i}\right)}{\tilde{m}\left(M_{i}-M_{j}\right)}=-\frac{c_{4}^{(2)}}{c^{(1)}} \times \begin{cases}\operatorname{Re}\left(h_{e}\right)_{i j} & \mathrm{NH} \\ \operatorname{Re}\left(\tilde{I} h_{e} \tilde{I}^{T}\right)_{i j} & \mathrm{IH} .\end{cases}
$$

Using the above result in the expression of $\epsilon_{i \alpha}$ (along with $\left.\left(\lambda_{\nu}^{0}\right)_{i \alpha}\left(\lambda_{\nu}^{0}\right)_{\alpha j}^{\dagger}\right)$, one obtains for $\mathrm{NH}$ :

$$
\epsilon_{i \alpha}^{N H}=-\frac{c_{4}^{(2)}}{c^{(1)}} \sum_{\beta=e, \mu, \tau}\left(\frac{m_{\beta}}{v}\right)^{2} \sum_{j \neq i}\left\{F^{(j, i)} \frac{m_{j}}{\tilde{m}} \operatorname{Re}\left(U_{\beta i}^{*} U_{\beta j}\right) \operatorname{Im}\left(U_{\alpha i}^{*} U_{\alpha j}\right)\right\},
$$

while for IH:

$$
\epsilon_{i \alpha}^{I H}=-\frac{c_{4}^{(2)}}{c^{(1)}} \sum_{\beta=e, \mu, \tau}\left(\frac{m_{\beta}}{v}\right)^{2} \sum_{j \neq i} \sum_{m, n}\left\{F^{(j, i)} \frac{m_{n}}{\tilde{m}} \tilde{I}_{i m} \tilde{I}_{j n} \operatorname{Re}\left(U_{\beta m}^{*} U_{\beta n}\right) \operatorname{Im}\left(U_{\alpha m}^{*} U_{\alpha n}\right)\right\} .
$$


In the above expressions the dominant term arises when $\beta=\tau$ in the sum over charged lepton flavours. Keeping only $\beta=\tau$ and defining

$$
\Phi_{i j}^{(\alpha)} \equiv \operatorname{Re}\left(U_{\tau i}^{*} U_{\tau j}\right) \operatorname{Im}\left(U_{\alpha i}^{*} U_{\alpha j}\right)
$$

the asymmetries read:

$$
\begin{aligned}
\epsilon_{1 \alpha}^{N H} & =-\frac{c_{4}^{(2)}}{c^{(1)}}\left(\frac{m_{\tau}}{v}\right)^{2}\left[F^{(2,1)} \frac{m_{2}}{\tilde{m}} \Phi_{12}^{(\alpha)}+F^{(3,1)} \frac{m_{3}}{\tilde{m}} \Phi_{13}^{(\alpha)}\right] \\
\epsilon_{2 \alpha}^{N H} & =+\frac{c_{4}^{(2)}}{c^{(1)}}\left(\frac{m_{\tau}}{v}\right)^{2}\left[F^{(1,2)} \frac{m_{1}}{\tilde{m}} \Phi_{12}^{(\alpha)}-F^{(3,2)} \frac{m_{3}}{\tilde{m}} \Phi_{23}^{(\alpha)}\right] \\
\epsilon_{3 \alpha}^{N H} & =+\frac{c_{4}^{(2)}}{c^{(1)}}\left(\frac{m_{\tau}}{v}\right)^{2}\left[F^{(1,3)} \frac{m_{1}}{\tilde{m}} \Phi_{13}^{(\alpha)}+F^{(2,3)} \frac{m_{2}}{\tilde{m}} \Phi_{23}^{(\alpha)}\right] .
\end{aligned}
$$

The IH case is obtained by a straightforward permutation of indices in the $m_{j}$ and $\Phi_{i j}^{\alpha}$ factors. We are now in a position to identify the dependence of the baryon asymmetry on the low-energy $\mathrm{CP}$ violating phases, contained in the factors $\Phi_{i j}^{(\alpha)}$. In the case of $\mathrm{NH}$, only $\Phi_{i j}^{(e)}$ are relevant, as the $e$ flavour is the least washed-out one:

$$
\begin{aligned}
\Phi_{12}^{(e)} & =\frac{1}{2} s_{12}^{2} c_{12}^{2} s_{23}^{2} c_{13}^{2} \sin \left(\alpha_{1}-\alpha_{2}\right)+\mathcal{O}\left(s_{13}^{2}\right) \\
\Phi_{13}^{(e)} & =s_{12} c_{12} s_{23} c_{23} c_{13}^{2} s_{13} \cos \left(\frac{\alpha_{1}}{2}\right) \sin \left(\frac{\alpha_{1}}{2}+\delta\right)+\mathcal{O}\left(s_{13}^{2}\right) \\
\Phi_{23}^{(e)} & =s_{12} c_{12} s_{23} c_{23} c_{13}^{2} s_{13} \cos \left(\frac{\alpha_{2}}{2}\right) \sin \left(\frac{\alpha_{2}}{2}+\delta\right)+\mathcal{O}\left(s_{13}^{2}\right) .
\end{aligned}
$$

For IH, all flavours are roughly equally washed out, so one needs also $\Phi_{i j}^{(\mu, \tau)}$. Expanding to first non-trivial order in $s_{13}$ we find:

$$
\begin{aligned}
\Phi_{12}^{(\mu)} & =-\frac{1}{2} s_{12}^{2} c_{12}^{2} s_{23}^{2} c_{23}^{2} \sin \left(\alpha_{1}-\alpha_{2}\right) \\
\Phi_{13}^{(\mu)} & =\frac{1}{2} s_{12}^{2} s_{23}^{2} c_{23}^{2} c_{13}^{2} \sin \alpha_{1} \\
\Phi_{23}^{(\mu)} & =\frac{1}{2} c_{12}^{2} s_{23}^{2} c_{23}^{2} c_{13}^{2} \sin \alpha_{2} \\
\Phi_{12}^{(\tau)} & =-\frac{1}{2} s_{12}^{2} c_{12}^{2} s_{23}^{4} \sin \left(\alpha_{1}-\alpha_{2}\right) \\
\Phi_{13}^{(\tau)} & =-\frac{1}{2} s_{12}^{2} s_{23}^{2} c_{23}^{2} c_{13}^{2} \sin \alpha_{1} \\
\Phi_{23}^{(\tau)} & =-\frac{1}{2} c_{12}^{2} s_{23}^{2} c_{23}^{2} c_{13}^{2} \sin \alpha_{2}
\end{aligned}
$$

The above expressions explain well the two main qualitative features of this scenario:

- In order to have a lepton asymmetry which does not vanish in the limit $s_{13} \rightarrow 0$ it is necessary to have non-vanishing Majorana phases.

- In the limit $\alpha_{1}=\alpha_{2}=0$ the lepton asymmetry is proportional to $s_{13} \sin \delta$ but the overall scale is substantially smaller than in the generic case with non-vanishing Majorana phases. 

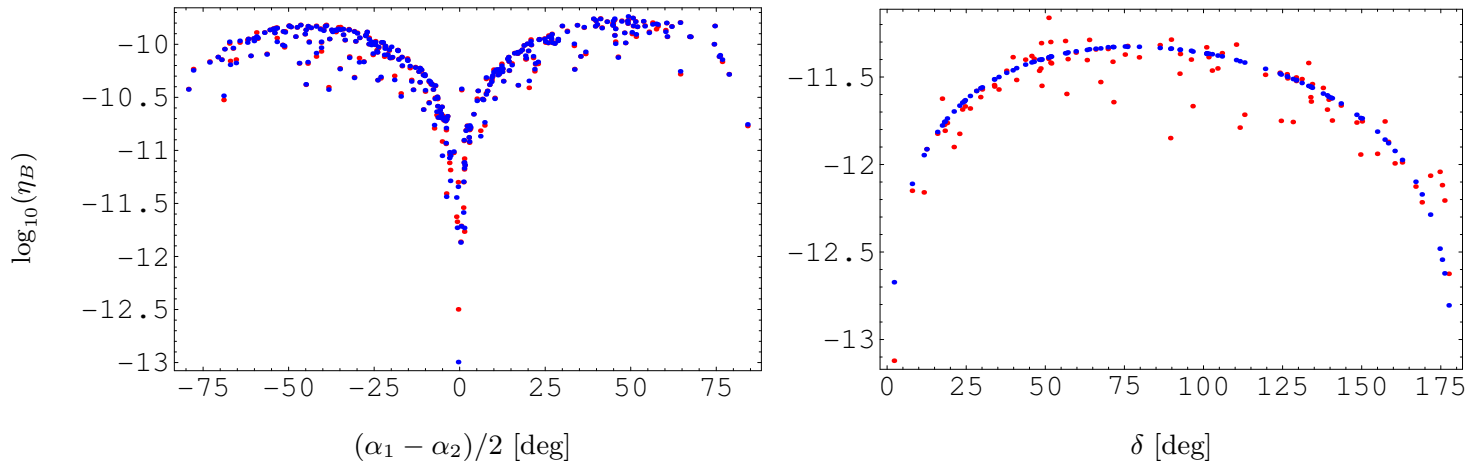

Figure 5: Left: $\eta_{B}$ vs. $\left(\alpha_{1}-\alpha_{2}\right) / 2$ varying $m_{1} \leq 10^{-2} \mathrm{eV}, s_{13} \leq 0.1$, and $0<\delta<180^{\circ}$ in the NH scenario. Right: $\eta_{B}$ vs. $\delta$ for $\alpha_{1}=\alpha_{2}=0$, setting $m_{1}=10^{-3} \mathrm{eV}$ and $s_{13}=0.03$ in the NH scenario. In both plots $c_{4}^{(2)}=10^{-2}, c^{(1)}=10^{-1}, M_{\nu}=10^{9} \mathrm{GeV}$, and red (blue) points correspond to the inclusion (exclusion) of quantum effects.
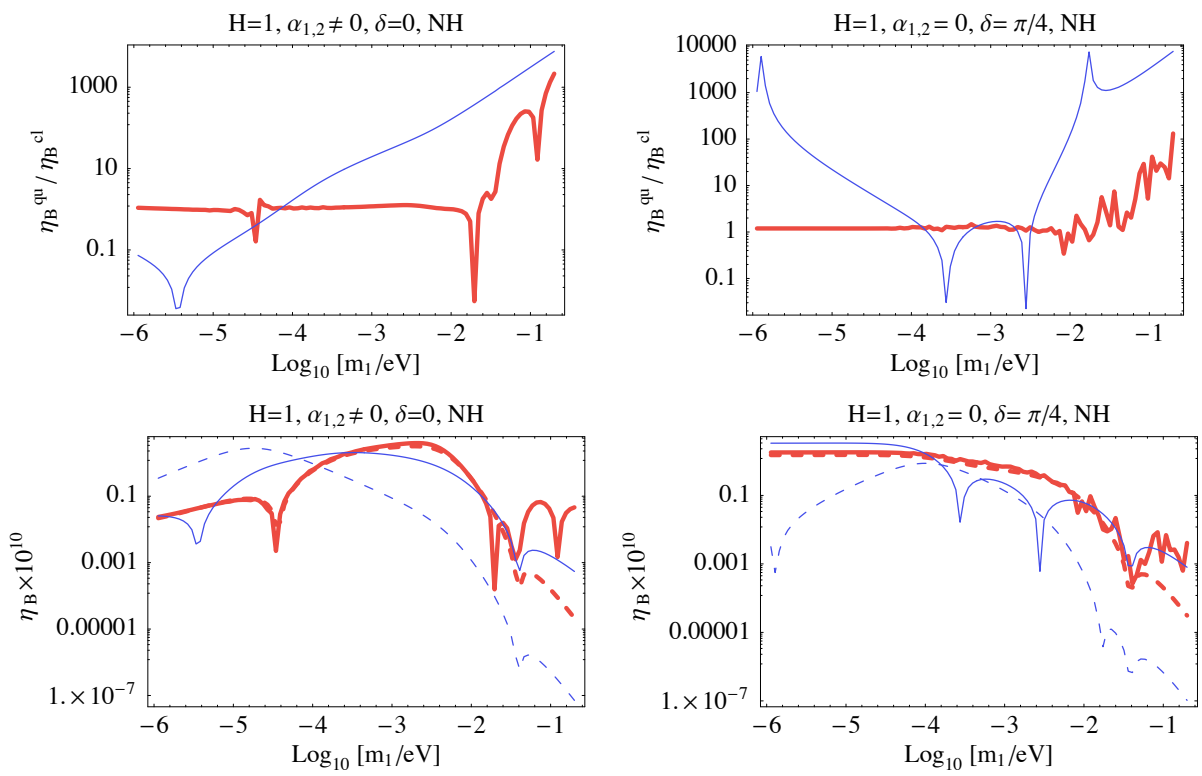

Figure 6: Dependence on $m_{1}$, in normal hierarchy and $H=1$. Thick red and thin blue lines correspond to $c^{(1)}=6 \times 10^{-3}$ and $c^{(1)}=2 \times 10^{-5}$, respectively; the other RH mass splitting coefficients are taken to be: $c_{1,2,3}^{(2)}=0$ and $c_{4}^{(2)}=0.1 c^{(1)}$. The PMNS phases and $s_{13}$ are chosen to be $\alpha_{1}=36^{\circ}, \alpha_{2}=60^{\circ}, \delta=0, s_{13}=10^{-3}$ and $\alpha_{1}=\alpha_{2}=0, \delta=\pi / 4, s_{13}=0.1$. Top: The absolute value of the ratio of the baryon asymmetry with quantum effects $\left(\eta_{B}^{\mathrm{qu}}\right)$ and without quantum effects $\left(\eta_{B}^{\mathrm{cl}}\right)$. Bottom: The absolute values of $\eta_{B}^{\mathrm{qu}}$ (solid lines) and $\eta_{B}^{\mathrm{cl}}$ (dashed lines).

Qualitatively, these two observations agree with the conclusions obtained in Ref. 23 by means of a numerical study ${ }^{4}$ They are also qualitatively confirmed by our numerical evaluation of the

\footnotetext{
${ }^{4}$ A precise numerical comparison with Ref. 23 is not possible given the different assumptions about the structure of the $c_{j}^{(i)}$.
} 

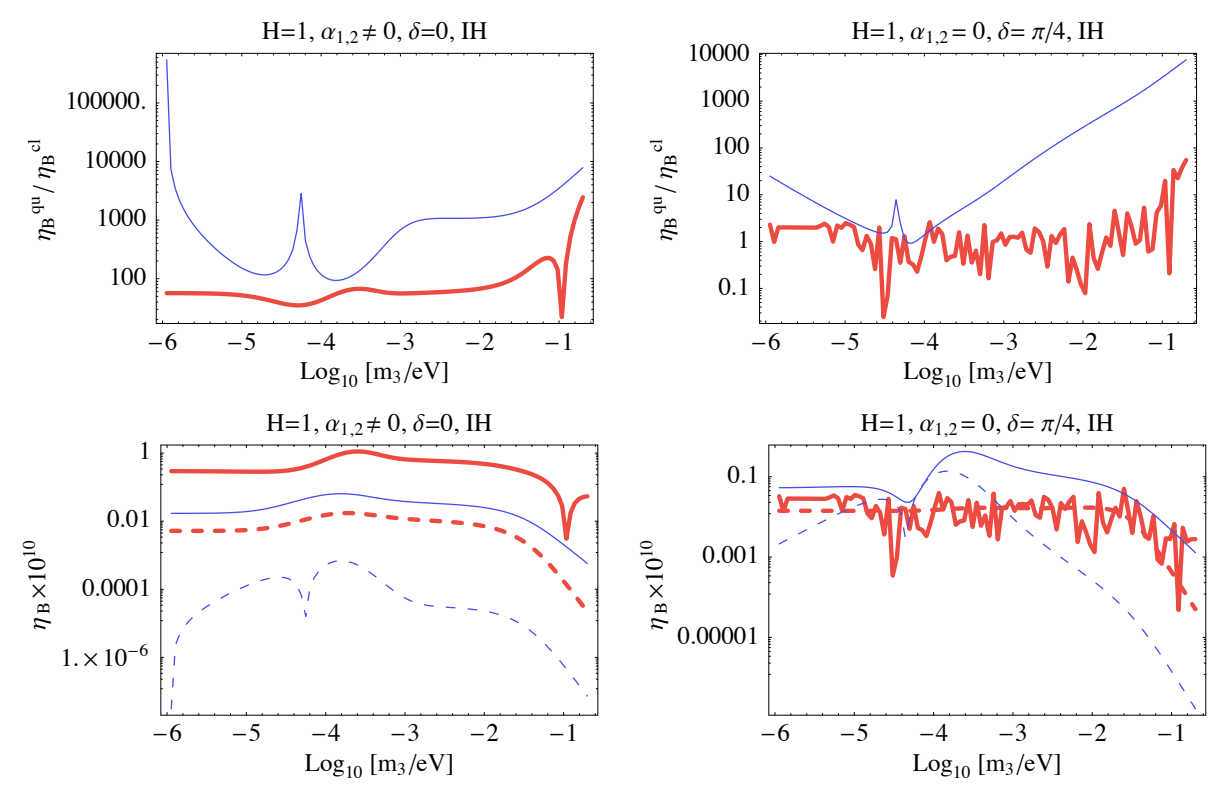

Figure 7: Dependence on $m_{3}$, in inverse hierarchy. Thick red and thin blue lines correspond to $c^{(1)}=6 \times 10^{-3}$ and $c^{(1)}=2 \times 10^{-5}$, respectively; the other RH mass splitting coefficients are taken to be: $c_{1,2,3}^{(2)}=0$ and $c_{4}^{(2)}=0.1 c^{(1)}$. The PMNS phases and $s_{13}$ are chosen to be $\alpha_{1}=36^{\circ}, \alpha_{2}=60^{\circ}, \delta=0, s_{13}=10^{-3}$ and $\alpha_{1}=\alpha_{2}=0, \delta=\pi / 4, s_{13}=0.1$. Top: The absolute value of the ratio of the baryon asymmetry with quantum effects $\left(\eta_{B}^{\mathrm{qu}}\right)$ and without quantum effects $\left(\eta_{B}^{\mathrm{cl}}\right)$. Bottom: The absolute values of $\eta_{B}^{\mathrm{qu}}$ (solid lines) and $\eta_{B}^{\mathrm{cl}}$ (dashed lines).

baryon asymmetry taking into account quantum effects, as shown in Fig. 5 .

It should be stressed that the above observations are only the main qualitative features and do not describe in detail all the allowed possibilities. In particular, the complete numerical study shows that the $\eta_{B} \propto s_{13} \sin \delta$ behaviour in the $\alpha_{1}=\alpha_{2}=0$ limit, derived from a linear expansion in $s_{13}$ and in the approximation $K_{e} \ll K_{\mu, \tau}$, is a good approximation only for $s_{13} \ll 0.1$ and small $m_{1}$. For large $s_{13}$ and large $m_{1}$ values the $\mathcal{O}\left(s_{13}^{2}\right)$ terms generate a sizable correction to the formally leading $s_{13} \sin \delta$ term. As a result, the asymmetry assumes a form of the type $\eta_{B} \propto \sin \delta(1+b \cos \delta)$, with $b=\mathcal{O}(1)$.

As far as the size of the baryon asymmetry is concerned, if the Majorana phases are large one can easily reach values of $\eta_{B}$ which are consistent with the experimental observations (see Fig. 5left). This is not possible if the Majorana phases are set to zero, at least in the single-Higgs scenario considered so far. In this case $\eta_{B}$ turns out to be smaller than the experimental value by at least one order of magnitude. However, the overall normalization of $\eta_{B}$ changes if we consider a two-Higgs doublet scenario (such as the Higgs sector of the MSSM), where the charged-lepton Yukawa couplings are enhanced by $\tan \beta=\left\langle H_{U}\right\rangle /\left\langle H_{D}\right\rangle>1$. In this case $\eta_{B} \propto \tan ^{2} \beta$ and the overall normalization can easily reach the experimental value even if the Majorana phases are set to zero. The MLFV framework with $H=I$, vanishing Majorana phases and large $\tan \beta$, provides a concrete scenario where the observed baryon asymmetry is directly linked to the measurable $\mathrm{CP}$ violating phase of the low-energy neutrino mass matrix.

We conclude that the impact of quantum effects in the $H=I$ case is qualitatively very similar 
to the $H \neq I$ scenario: memory effects become sizable for small splitting (in particular small $\left.c^{(1)}\right)$ and in the limit of light degenerate neutrinos. An illustration of the impact of these effects is provided by the the plots in Fig. 6 6. As can be noted, the effects can be quite dramatic, especially for IH. However, it should also be stressed that the largest relative impact is obtained in regions of the parameter space where $\eta_{B}$ is well below the experimental value.

\section{Conclusions}

In this paper we have studied resonant leptogenesis in the MLFV framework where it is assumed that the charged-lepton and the neutrino Yukawa couplings are the only irreducible sources of lepton-flavour symmetry breaking. In such a framework, the heavy RH neutrinos are highly degenerate in mass and their decays in the early Universe may give rise to the observed baryon asymmetry through the mechanism of resonant leptogenesis. Previous studies on the viability of leptogenesis in the MLFV framework [21, 22, 23] have been based on the assumption that classical Boltzmann equations suffice to analyse the dynamical generation of the baryon asymmetry. However, it has been recently shown [24, 26] that quantum Boltzmann equations are a more appropriate tool to study such a dynamics when the heavy RH neutrinos are degenerate in mass. Indeed, the quantum Boltzmann equations obtained starting from the non-equilibrium quantum field theory reveal that the CP asymmetry is a time-dependent oscillatory function which reduces to the value obtained in the classical approach only if the oscillation time is much larger than the interaction time. In resonant leptogensis this is not the case.

We have shown both analytically and numerically that neglecting the time dependence of the $\mathrm{CP}$ asymmetry may underestimate the baryon asymmetry by several orders of magnitude when a strong degeneracy among heavy $\mathrm{RH}$ neutrinos and small mass splittings in the light neutrino sectors are present. This is true both when the $\mathrm{CP}$ phases come from the $\mathrm{RH}$ sector (phases in the matrix $H)$ and when they come entirely from the left-handed sector $(H=I)$ and may be identified with the low energy PMNS phases.

\section{Acknowledgements}

The work of ADS is supported in part by the INFN "Bruno Rossi" Fellowship and in part by the US Department of Energy (DOE) under cooperative research agreement DE-FG02- 05ER41360. The work of GI is supported in part by the EU Contract No. MRTN-CT-2006-035482 FLAVIAnet. The work of AR is supported in part by the European Programmes "The Quest For Unification", contract MRTN-CT-2004-503369 and "UniverseNet", contract MRTN-CT-2006-035863.

\section{A Conventions for Boltzmann equations}

In the early Universe the quantum numbers conserved by sphaleron interactions are the $\Delta_{\alpha}=$ $B / 3-L_{\alpha}$. The pair of Boltzmann equations describing the generation of the baryon asymmetry are

$$
\frac{d Y_{N_{i}}}{d z}=-D_{i}\left(Y_{N_{i}}-Y_{N_{i}}^{e q}\right),
$$




$$
\frac{d Y_{\Delta_{\alpha}}}{d z}=-\sum_{i} \epsilon_{i \alpha} D_{i}\left(Y_{N_{i}}-Y_{N_{i}}^{e q}\right)-W_{\alpha}\left|A_{\alpha \alpha}\right| Y_{\Delta_{\alpha}}
$$

where at equilibrium the $N_{i}$ number density normalised to the entropy density of the universe is $Y_{N_{i}}^{e q}=z_{i}^{2} \mathcal{K}_{2}\left(z_{i}\right) /\left(4 g_{*}\right)$, where $z_{i}=z \sqrt{x_{i}}, g_{*}=106.75$ and $\mathcal{K}_{2}\left(z_{i}\right)$ is a modified Bessel function of the second kind. The washout parameters are generically defined by

$$
K_{i \alpha} \equiv \frac{\Gamma\left(N_{i} \rightarrow \ell_{\alpha} \bar{H}\right)}{H\left(T=M_{i}\right)}
$$

and we also make use of the quantities: $K_{\alpha}=\sum_{i} K_{i \alpha}$ and $K_{i}=\sum_{\alpha} K_{i \alpha}$. In terms of the parameters of the model, $K_{i \alpha}=\left|\left(\lambda_{\nu}\right)_{i \alpha}\right|^{2} v^{2} /\left(M_{i} m_{*}\right)$ with $m_{*} \approx 10^{-3} \mathrm{eV}$. The decay and washout terms appearing in the Boltzmann equations are

$$
D_{i}=K_{i} x_{i} z \frac{\mathcal{K}_{1}\left(z_{i}\right)}{\mathcal{K}_{2}\left(z_{i}\right)}, \quad W_{\alpha}=\sum_{i} \frac{1}{4} K_{i \alpha} \sqrt{x_{i}} \mathcal{K}_{1}\left(z_{i}\right) z_{i}^{3},
$$

while the matrix $\mathrm{A}$ is given by

$$
A=-\operatorname{diag}(151 / 179,344 / 537,344 / 537), \quad \text { for } \quad M_{1} \lesssim 10^{9} \mathrm{GeV} .
$$

For $10^{9} \mathrm{GeV} \lesssim M_{1} \lesssim 10^{12} \mathrm{GeV}$ and $M_{1} \gtrsim 10^{12} \mathrm{GeV}$, the two-flavour and the one-flavour regimes should be applied, respectively [29].

Finally,

$$
Y_{B}=\frac{12}{37} \sum_{\alpha} Y_{\Delta_{\alpha}}(z \rightarrow \infty)
$$

to be compared with the measured value $Y_{B}=(8.7 \pm 0.3) \times 10^{-11}$ or with the baryon asymmetry normalized with respect to the photon number density (instead of the entropy density) $\eta_{B}=$ $(6.3 \pm 0.3) \times 10^{-10}$.

\section{B Analysis of CP violating weak-basis invariants}

The independent CP-violating phases of the model can be characterised in terms of weak-basis invariants, i.e. quantities that are insensitive to changes of basis or re-phasing of the lepton fields. The MLFV scenario under investigation has six independent CPV invariants coming from the Yukawa sector.

The simplest necessary conditions for $\mathrm{CP}$ invariance can be cast in the following weak-basis invariant form 32

$$
\begin{aligned}
B_{1} & \equiv \operatorname{Im} \operatorname{Tr}\left[h_{\nu}\left(M_{\nu}^{\dagger} M_{\nu}\right) M_{\nu}^{*} h_{\nu}^{*} M_{\nu}\right]=0, \\
B_{2} & \equiv \operatorname{Im} \operatorname{Tr}\left[h_{\nu}\left(M_{\nu}^{\dagger} M_{\nu}\right)^{2} M_{\nu}^{*} h_{\nu}^{*} M_{\nu}\right]=0, \\
B_{3} & \equiv \operatorname{Im} \operatorname{Tr}\left[h_{\nu}\left(M_{\nu}^{\dagger} M_{\nu}\right)^{2} M_{\nu}^{*} h_{\nu}^{*} M_{\nu}\left(M_{\nu}^{\dagger} M_{\nu}\right)\right]=0,
\end{aligned}
$$

\footnotetext{
${ }^{5}$ We used the approximation in which $A$ is a diagonal matrix. For the full expression see refs. 27, 28, 29,
} 
where $h_{\nu}=\lambda_{\nu} \lambda_{\nu}^{\dagger}$ and $M_{\nu}$ denotes a generic heavy neutrino mass term. The invariants $B_{1,2,3}$ are independent and survive in the limit $\lambda_{e} \rightarrow 0$. One can construct three other independent invariants that explicitly ${ }^{6}$ involve $\lambda_{e}$ by simply replacing one $h_{\nu}$ entry in $B_{1,2,3}$ with $h_{e} \equiv \lambda_{\nu} \lambda_{e}^{\dagger} \lambda_{e} \lambda_{\nu}^{\dagger}$ :

$$
\begin{aligned}
\tilde{B}_{1} & \equiv \operatorname{Im} \operatorname{Tr}\left[h_{\nu}\left(M_{\nu}^{\dagger} M_{\nu}\right) M_{\nu}^{*} h_{e}^{*} M_{\nu}\right]=0, \\
\tilde{B}_{2} & \equiv \operatorname{Im} \operatorname{Tr}\left[h_{\nu}\left(M_{\nu}^{\dagger} M_{\nu}\right)^{2} M_{\nu}^{*} h_{e}^{*} M_{\nu}\right]=0, \\
\tilde{B}_{3} & \equiv \operatorname{Im} \operatorname{Tr}\left[h_{\nu}\left(M_{\nu}^{\dagger} M_{\nu}\right)^{2} M_{\nu}^{*} h_{e}^{*} M_{\nu}\left(M_{\nu}^{\dagger} M_{\nu}\right)\right]=0 .
\end{aligned}
$$

Note that $B_{1,2,3}$ and $\tilde{B}_{1,2,3}$ are in direct correspondence with (linearly independent combinations of) the $\mathrm{CP}$ asymmetries $\epsilon_{\alpha}^{(j, i)}$ relevant for flavoured leptogenesis. In particular, $B_{1,2,3}$ correspond to the combinations relevant for the 1-flavour regime. This can be seen by working in the weak-basis where $M_{\nu}$ and $\lambda_{e}$ are diagonal. In this basis, for example, $B_{1}$ and $\tilde{B}_{1}$ read:

$$
\begin{aligned}
B_{1} & =\sum_{i<j} M_{i} M_{j}\left(M_{j}^{2}-M_{i}^{2}\right) \sum_{\alpha=e, \mu, \tau} \operatorname{Im}\left[\left(\lambda_{\nu}\right)_{i \alpha}\left(\lambda_{\nu}^{\dagger}\right)_{\alpha j}\left(\lambda_{\nu} \lambda_{\nu}^{\dagger}\right)_{i j}\right] \\
\tilde{B}_{1} & =\sum_{i<j} M_{i} M_{j}\left(M_{j}^{2}-M_{i}^{2}\right) \sum_{\alpha=e, \mu, \tau} \frac{m_{\alpha}^{2}}{v^{2}} \operatorname{Im}\left[\left(\lambda_{\nu}\right)_{i \alpha}\left(\lambda_{\nu}^{\dagger}\right)_{\alpha j}\left(\lambda_{\nu} \lambda_{\nu}^{\dagger}\right)_{i j}\right] .
\end{aligned}
$$

If $M_{\nu}$ is proportional to the identity, the hermiticity of $h_{\nu, e}$ and the cyclic property of the trace operation imply that the $B_{i}, \tilde{B}_{i}$ vanish identically. The next step is to break the degeneracy of the heavy neutrinos in a way consistent with the MLFV hypothesis. Selecting for convenience the 0-superscript basis, let us now investigate under which conditions on $M_{\nu}$ of Eq. (6) and $\lambda_{\nu}^{0}$ of Eq.(8) the $B_{i}, \tilde{B}_{i}$ are non-vanishing.

- If we confine ourselves to terms quadratic in the Yukawa couplings, i.e. proportional to $c^{(1)}$, then we have that:

- $B_{i}=0$ because of the hermiticity of $h_{\nu}^{0}$ and properties of the trace operator. This implies that in the unflavoured regime the $\mathrm{CP}$ asymmetries vanish;

- $\tilde{B}_{i} \neq 0$ as long as $H \neq I$. Since $\tilde{B}_{i} \propto c^{(1)} \operatorname{Im} \operatorname{Tr}\left[\left(h_{\nu}^{0}\right)^{a} h_{e}^{*}\right]=0$ ( $a$ is some integer $)$ and the restriction $H=I$ implies that $h_{\nu}^{0}$ is real, it turns out that leptogenesis is possible in the flavoured regime only if $H \neq I$.

- If we include those terms in $M_{R}$ that are quartic in the Yukawa couplings, i.e. proportional to $c_{1,2,3,4}^{(2)}$, then:

- $B_{i} \neq 0$ if any of the $c_{i}^{(2)} \neq 0$, as long as $H \neq I$. If $H=I$ then not only $h_{\nu}^{0}$ is real, but also $M_{\nu}$ and therefore $B_{1,2,3}=0$. So one concludes that with $H=I$ unflavoured leptogenesis is not viable;

- $\tilde{B}_{i} \neq 0$ if any of the $c_{i}^{(2)} \neq 0$. In this case, even setting $H=I$ leads to non-zero $\tilde{B}_{i}$ as long as $c_{4}^{(2)} \neq 0$. So one concludes that leptogenesis with $H=I$ is potentially viable only in the flavoured regime with $c_{4}^{(2)} \neq 0$.

\footnotetext{
${ }^{6}$ Of course $\lambda_{e}$ can also appear implicitly through the contributions to $M_{\nu}$ allowed by MLFV.
} 
In conclusion, flavour effects open at least in principle two new regimes for MLFV-leptogenesis which are not allowed in the 1-flavour limit: (i) the case in which RH mass splitting is induced only (or mainly) by $c^{(1)}$. This situation requires $H \neq I$, namely $\mathrm{CPV}$ in the $\mathrm{RH}$ sector; (ii) the case in which CPV arises only from PMNS phases, namely $H=I$ and $M_{\nu}$ is real.

\section{References}

[1] D. N. Spergel et al. [WMAP Collaboration], arXiv:astro-ph/0603449.

[2] M. Fukugita and T. Yanagida, Phys. Lett. B 174, 45 (1986).

[3] G. F. Giudice, A. Notari, M. Raidal, A. Riotto and A. Strumia, Nucl. Phys. B 685, 89 (2004) arXiv:hep-ph/0310123 ; W. Buchmuller, P. Di Bari and M. Plumacher, Annals Phys. 315 (2005) 305 arXiv:hep-ph/0401240].

[4] A partial list: W. Buchmuller, P. Di Bari and M. Plumacher, Nucl. Phys. B 643 (2002) 367 arXiv:hep-ph/0205349]; J. R. Ellis, M. Raidal and T. Yanagida, Phys. Lett. B 546 (2002) 228 arXiv:hep-ph/0206300; G. C. Branco, R. Gonzalez Felipe, F. R. Joaquim and M. N. Rebelo, Nucl. Phys. B 640 (2002) 202 arXiv:hep-ph/0202030]; G. C. Branco, R. Gonzalez Felipe, F. R. Joaquim, I. Masina, M. N. Rebelo and C. A. Savoy, Phys. Rev. D 67, 073025 (2003) arXiv:hep-ph/0211001); R. N. Mohapatra, S. Nasri and H. B. Yu, Phys. Lett. B 615 (2005) 231 [arXiv:hep-ph/0502026]; A. Broncano, M. B. Gavela and E. Jenkins, Nucl. Phys. B 672 (2003) 163 |arXiv:hep-ph/0307058]; A. Pilaftsis, Phys. Rev. D 56 (1997) 5431 arXiv:hep-ph/9707235); E. Nezri and J. Orloff, JHEP 0304 (2003) 020 arXiv:hepph/0004227; S. Davidson and A. Ibarra, Nucl. Phys. B 648, 345 (2003) arXiv:hepph/0206304]; S. Davidson, JHEP 0303 (2003) 037 [arXiv:hep-ph/0302075]; S. T. Petcov, W. Rodejohann, T. Shindou and Y. Takanishi, Nucl. Phys. B 739 (2006) 208 arXiv:hepph/0510404.

[5] P. Minkowski, Phys. Lett. B 67 (1977) 421; M. Gell-Mann, P. Ramond and R. Slansky, Proceedings of the Supergravity Stony Brook Workshop, New York 1979, eds. P. Van Nieuwenhuizen and D. Freedman; T. Yanagida, Proceedinds of the Workshop on Unified Theories and Baryon Number in the Universe, Tsukuba, Japan 1979, ed.s A. Sawada and A. Sugamoto; R. N. Mohapatra, G. Senjanovic, Phys.Rev.Lett. 44 (1980)912.

[6] A.D. Sakharov. JETP Lett. 5 (1967) 24.

[7] For a review, see A. Riotto and M. Trodden, Ann. Rev. Nucl. Part. Sci. 49, 35 (1999).

[8] V.A. Kuzmin, V.A. Rubakov, and M.E. Shaposhnikov. Phys. Lett., B155:36, 1985.

[9] S. Davidson and A. Ibarra, Phys. Lett. B 535, 25 (2002).

[10] A. Abada, S. Davidson, F. X. Josse-Michaux, M. Losada and A. Riotto, JCAP 0604, 004 (2006).

[11] S. Antusch and A. M. Teixeira, JCAP 0702, 024 (2007).

[12] F. R. Joaquim, I. Masina and A. Riotto, arXiv:hep-ph/0701270

[13] For a recent review, see T. Moroi, AIP Conf. Proc. 805, 37 (2006). 
[14] M. Flanz, E. A. Paschos and U. Sarkar, Phys. Lett. B 345 (1995) 248 [Erratum-ibid. B 382 (1996) 447] [arXiv:hep-ph/9411366]; L. Covi and E. Roulet, Phys. Lett. B 399, 113 (1997) arXiv:hep-ph/9611425]; A. Pilaftsis, Phys. Rev. D 56, 5431 (1997) arXiv:hepph/9707235; T. Hambye, Nucl. Phys. B 633, 171 (2002) arXiv:hep-ph/0111089; A. Pilaftsis and T. E. J. Underwood, Nucl. Phys. B 692, 303 (2004) arXiv:hep-ph/0309342; A. Pilaftsis and T. E. J. Underwood, Phys. Rev. D 72, 113001 (2005) [arXiv:hep-ph/0506107].

[15] R. S. Chivukula and H. Georgi, Phys. Lett. B 188 (1987) 99; L. J. Hall and L. Randall, Phys. Rev. Lett. 65 (1990) 2939.

[16] G. D'Ambrosio, G. F. Giudice, G. Isidori and A. Strumia, Nucl. Phys. B 645 (2002) 155 arXiv:hep-ph/0207036.

[17] V. Cirigliano, B. Grinstein, G. Isidori and M. B. Wise, Nucl. Phys. B 728 (2005) 121 arXiv:hep-ph/0507001.

[18] V. Cirigliano and B. Grinstein, Nucl. Phys. B 752 (2006) 18 arXiv:hep-ph/0601111].

[19] B. Grinstein, V. Cirigliano, G. Isidori and M. B. Wise, Nucl. Phys. B 763 (2007) 35 arXiv:hep-ph/0608123.

[20] S. Davidson and F. Palorini, Phys. Lett. B 642 (2006) 72.

[21] V. Cirigliano, G. Isidori and V. Porretti, Nucl. Phys. B 763 (2007) 228 arXiv:hep$\mathrm{ph} / 0607068$.

[22] G. C. Branco, A. J. Buras, S. Jager, S. Uhlig and A. Weiler, JHEP 0709 (2007) 004 arXiv:hep-ph/0609067.

[23] S. Uhlig, arXiv:hep-ph/0612262.

[24] A. De Simone and A. Riotto, JCAP 0708 (2007) 002 arXiv:hep-ph/0703175].

[25] W. Buchmuller and S. Fredenhagen, Phys. Lett. B 483, 217 (2000) arXiv:hep-ph/0004145.

[26] A. De Simone and A. Riotto, JCAP 0708 (2007) 013 arXiv:0705.2183 [hep-ph].

[27] R. Barbieri, P. Creminelli, A. Strumia and N. Tetradis, Nucl. Phys. B 575 (2000) 61; T. Endoh, T. Morozumi and Z. h. Xiong, Prog. Theor. Phys. 111 (2004) 123;

[28] A. Abada, S. Davidson, F. X. Josse-Michaux, M. Losada and A. Riotto, JCAP 0604, 004 (2006); E. Nardi, Y. Nir, E. Roulet and J. Racker, JHEP 0601, 164 (2006).

[29] A. Abada, S. Davidson, A. Ibarra, F. X. Josse-Michaux, M. Losada and A. Riotto, JHEP 0609, 010 (2006).

[30] S. Blanchet and P. Di Bari, JCAP 0703, 018 (2007); S. Antusch, S. F. King and A. Riotto, JCAP 0611, 011 (2006); S. Pascoli, S. T. Petcov and A. Riotto, . Rev. D 75, 083511 (2007); G. C. Branco, R. Gonzalez Felipe and F. R. Joaquim, Phys. Lett. B 645 (2007) 432; S. Pascoli, S. T. Petcov and A. Riotto; . Rev. D 75, 083511 (2007); G. Engelhard, Y. Grossman, E. Nardi and Y. Nir, hep-ph/0612187; S. Blanchet, P. Di Bari and G. G. Raffelt, JCAP 0703, 012 (2007); S. Pascoli, S. T. Petcov and A. Riotto, Nucl. Phys. B 774, 1 (2007); A. De Simone and A. Riotto, JCAP 0702 (2007) 005; T. Shindou and T. Yamashita, JHEP 0709, 043 (2007); F. X. Josse-Michaux and A. Abada, arXiv:hep-ph/0703084. 
[31] J. A. Casas and A. Ibarra, Nucl. Phys. B 618 (2001) 171 arXiv:hep-ph/0103065.

[32] G. C. Branco, T. Morozumi, B. M. Nobre and M. N. Rebelo, Nucl. Phys. B 617, 475 (2001) arXiv:hep-ph/0107164. 